\title{
Low emittance lattice design from first principles: Reverse bending and longitudinal gradient bends
}

\author{
B. Riemann ${ }^{*}$ and A. Streun ${ }^{\dagger}$ \\ Paul Scherrer Institut, CH-5232 Villigen PSI, Switzerland
}

(Received 29 October 2018; published 5 February 2019)

\begin{abstract}
A unit cell with one homogeneous bending magnet is commonly used in the design of multi-bend achromat (MBA) lattices for the new generation of diffraction limited storage rings. But significantly lower emittance at moderate focusing properties can be achieved by combining longitudinal gradient bends (LGB) and reverse bends (RB) in a periodic lattice unit cell. LGBs alone, however, are of rather limited gain. We investigate the emittance achievable for different unit cell classes as a function of the cell phase advance in a general framework, i.e., with a minimum of assumptions on the particular cell optics. Each case is illustrated with a practical example of a realistic lattice cell, eventually leading to the LGB/RB unit cell of the baseline lattice for the upgrade of the Swiss Light Source.
\end{abstract}

DOI: 10.1103/PhysRevAccelBeams.22.021601

\section{INTRODUCTION}

The quantum nature of light is the origin of finite emittance in an electron storage ring: sudden loss of energy due to photon emission causes an electron to start a betatron oscillation around the closed orbit corresponding to its reduced energy. The orbit position as a function of energy is given by the lattice dispersion. Thus emittance is minimized by suppression of dispersion at locations where radiation is emitted, i.e., the bending magnets (bends). In a planar, separate-function lattice, this can be done in three ways: (1) Horizontal focusing of the beam into the bends, since dispersion occurs in the horizontal dimension in a planar lattice. (2) Using many bends of small deflection angle to limit the dispersion growth inside the bend. This is the concept of the multibend achromat (MBA) lattice in its classic form [1,2] and its modifications (hybrid MBA) [3]. (3) Variation of the magnetic field inside the bend to compensate the growth of dispersion beyond the magnet center. This is the concept of the longitudinal gradient bend (LGB) [4]. For the new generation of diffraction limited storage rings, technological progress enabled miniaturization of vacuum chambers and magnets. This leads to a reduction of unit cell length, so that the double or triple bend achromats of third generation light sources could be replaced by MBAs containing five or more lattice cells

\footnotetext{
*bernard.riemann@psi.ch

andreas.streun@psi.ch
}

Published by the American Physical Society under the terms of the Creative Commons Attribution 4.0 International license. Further distribution of this work must maintain attribution to the author(s) and the published article's title, journal citation, and DOI. within the same arc length, as pioneered by MAX IV [5]. Since the emittance $\varepsilon$ scales with the inverse cube of unit cell bending angle [6,7], the introduction of small-aperture MBA lattices enabled emittance reduction by $1-2$ orders of magnitude compared to third generation light sources.

The emittance of a classic MBA is dominated by the emittance of the unit cell. The two dispersion suppressor cells at the ends of the arc are similar to half unit cells. The unit cell is made from a bend and focusing elements (i.e., quadrupoles) to provide periodic solutions for beta functions and dispersion in order to string together several cells.

The requirement of a horizontal focus in the bend center for minimizing dispersion leads to a high horizontal betatron phase advance $2 \phi$ of a low emittance cell. We consider phase advances $2 \phi<\pi$ as sensible, because solutions with $2 \phi>\pi$ require a second focus of the horizontal beta function, resulting in rather long cells.

The so-called theoretical minimum emittance (TME) cell provides the minimum emittance for a unit cell containing one homogeneous bend, as can be shown without any assumptions on the particular cell optics [8], but the corresponding phase advance is very high. Therefore most lattices are based on homogeneous-bend (HOM) cells at lower phase advance providing about three times larger emittance than the TME.

To further reduce the emittance, LGBs and reverse bends (RB, also called antibends) gained interest: LGBs have the potential to achieve sub-TME emittance by concentrating the quantum excitation in regions of vanishing dispersion, i.e., the bend center [9-11]. And (weak) RBs at the cell ends are useful to reduce the dispersion at the LGB center by manipulating the periodic solution of the dispersion function [12-14]. 
In this work we generalize the TME cell and the class of homogeneous-bend cells towards two different bends per cell with arbitrary longitudinal gradient but no transverse gradient, still not making any assumptions on the detailed focusing in the cell. This allows a general study of the alternative concept of the RB cell with and without LGB in a common framework.

It is shown that, while RB cells and LGB cells both may have slight advantages over HOM cells, only a combination of both (LGB/RB cell) enables superior emittance reduction and is also compatible with the requirements of classic MBAs built from periodic unit cells.

In the following, we first discuss unit cells with one bending magnet (Sec. II-IV) and then generalize to cells with two different bending magnets (Sec. V). General treatment of emittance, optimal parameters, and cell classes is done with a minimum of assumptions on the particular structure of the unit cell.

The design of a real cell, however, has to provide horizontal and vertical stability, has to take into account technical limitations and will strive for a minimum cell length. Therefore, the general treatment of each cell class is accompanied by the design [15] of a realistic example cell, which eventually cumulates in the present baseline design for the upgrade of the Swiss Light Source, SLS 2.0 [16-18] (Sec. VI).

\section{UNIT CELLS WITH ONE BEND}

We consider a half cell of unspecified length and focusing properties, the ends of which are denoted by indices $q \in\{0,1\}$. The half-cell ends are symmetry planes of optical functions (see Fig. 1).

The bending magnet at position 0 with full length $2 L_{0}$ generates a total bending angle $2 \theta_{0}>0$, which for cells with only one bend equals the bending angle per cell. As this angle is small $\left(\theta_{0} \ll 1\right)$, the effective focal length of the bending magnet is $f=L_{0} / \theta_{0}^{2} \gg L_{0}$ such that $\beta(s)$ approximately propagates like in a drift space, $\beta(s)=$ $\beta_{0}+s^{2} / \beta_{0}$-this is consistent with $[7,9,19]$ and confines

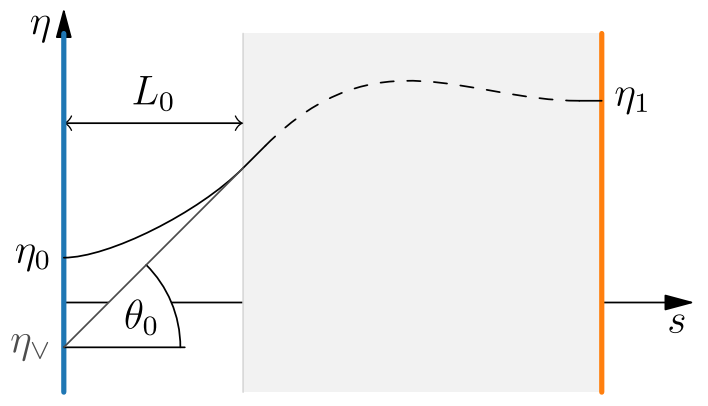

FIG. 1. Course of dispersion and equivalent thin-dipole dispersion for half-cells with one bend. The half-cell ends are denoted by blue and orange lines. In the bend-free region (shaded area), $\beta(s)$ and $\eta(s)$ are unspecified. our model to magnets without transverse gradients. Thus the phase advance in the bending magnet is always defined by $\beta_{0}$, resulting in a lower limit for the half-cell phase advance

$$
\phi>\arctan \left(L_{0} / \beta_{0}\right) \quad \text { or } \quad \beta_{0}>L_{0} \cot \phi .
$$

Unlike $\beta(s)$, the dispersion function $\eta(s)$ inside the bend depends specifically on the shape of its magnetic field, respectively the closed-orbit curvature $b(s)$, via [9]

$$
\eta(s)=\eta_{0}+\int_{0}^{s} \eta^{\prime}(\tilde{s}) \mathrm{d} \tilde{s}, \text { with } \eta^{\prime}(s)=\int_{0}^{s} b(\tilde{s}) \mathrm{d} \tilde{s} .
$$

The dispersion outside of the bending magnet depends only on its length and bending angle, as at its ends $\eta\left(L_{0}\right)=\eta\left(-L_{0}\right)$ and $\eta^{\prime}\left( \pm L_{0}\right)= \pm \theta_{0}$. It is therefore reasonable for matching purposes to introduce the equivalent dispersion value $\eta_{\vee}=\eta\left(L_{0}\right)-L_{0} \cdot \eta^{\prime}\left(L_{0}\right)$ at the bend center (position 0 ) that would occur if the bending magnet was thin, but retained its bending angle (Fig. 1); this method is also used in [20]. In case of a nonfocusing bend, we can apply partial integration and obtain

$$
\eta_{\vee}=\eta_{0}-\int_{0}^{L_{0}} s \cdot b(s) \mathrm{d} s .
$$

The horizontal transfer matrix of the half-cell [21] can be expressed as

$$
\mathbf{T}=\mathbf{B}_{1} \mathbf{R}(\phi) \mathbf{B}_{0}^{-1} \quad \text { with } \quad \mathbf{B}=\frac{1}{\sqrt{\beta}}\left(\begin{array}{cc}
\beta & 0 \\
-\alpha & 1
\end{array}\right),
$$

where $\mathbf{B}_{q}$ is the mapping from normalized phase space to standard phase space at the respective end $q$ of the half-cell, and $\mathbf{R}(\phi)$ is a clockwise rotation matrix with the half-cell phase advance $\phi$. The matching condition for the thindipole dispersion can then be written as

$$
\mathbf{B}_{1}^{-1}\left(\begin{array}{c}
\eta_{1} \\
0
\end{array}\right)=\mathbf{R}(\phi) \mathbf{B}_{0}^{-1}\left(\begin{array}{c}
\eta_{\vee} \\
\theta_{0}
\end{array}\right)
$$

Insertion of optics expressions for the $\mathbf{B}_{q}$ matrices using symmetry conditions $\left(\alpha_{q}=0\right)$ yields

$$
\begin{aligned}
& \vec{P}_{1}=\mathbf{R}(\phi) \vec{P}_{0}, \quad \text { with } \\
& \vec{P}_{0}=\frac{1}{\sqrt{\beta_{0}}}\left(\begin{array}{c}
\eta_{\vee} \\
\theta_{0} \beta_{0}
\end{array}\right), \quad \vec{P}_{1}=\left(\begin{array}{c}
\eta_{1} / \sqrt{\beta_{1}} \\
0
\end{array}\right) .
\end{aligned}
$$

This is shown in Fig. 2 with the half-cell phase advance

$$
\phi=\operatorname{atan} 2\left(\theta_{0} \beta_{0}, \eta_{\vee}\right),
$$


where atan 2 is the four-quadrant inverse tangent, returning the signed angle of a point $(x, y)$ in the Euclidean plane with the $x$ axis. It follows that

$$
\eta_{\vee}=\theta_{0} \beta_{0} \cot \phi
$$

The previously mentioned drift-space assumption for the bend implies the horizontal damping partition $J_{\mathrm{x}} \approx 1$ [6], so that the emittance contribution of a cell is proportional to the fraction of radiation integrals [22]

$$
\begin{gathered}
\varepsilon \propto \frac{I_{5}}{I_{2}} \quad \text { with } \quad I_{5}=\int\left|b^{3}(s)\right| \mathcal{H}(s) \mathrm{d} s, \\
I_{2}=\int b^{2}(s) \mathrm{d} s, \text { and } \mathcal{H}(s)=\gamma \eta^{2}+2 \alpha \eta \eta^{\prime}+\beta \eta^{\prime 2}
\end{gathered}
$$

being the dispersion action. Throughout the cell, the action of thin-dipole dispersion

$$
\mathcal{H}_{\mathrm{BF}}=\left|\vec{P}_{0}\right|^{2}=\frac{\eta_{v}^{2}}{\beta_{0}}+\theta_{0}^{2} \beta_{0}=\left|\vec{P}_{1}\right|^{2}
$$

is constant (Fig. 2), and thus $\mathcal{H}_{\mathrm{BF}}$ is also the dispersion action in the bend-free region.

\section{HOMOGENEOUS BEND}

For a homogeneous-bend cell (HOM cell) with curvature $b=\theta_{0} / L_{0}$, the fraction of radiation integrals from Eq. (9) simplifies to

$$
\frac{I_{5}}{I_{2}}=\frac{\theta_{0}}{L_{0}}\langle\mathcal{H}\rangle_{0}=\theta_{0}^{3}\langle\hat{\mathcal{H}}\rangle_{0}
$$

where $\langle\cdot\rangle_{0}$ denotes the average over the length of the bending magnet [22], and the normalized average of the dispersion action is defined via

$$
\langle\hat{\mathcal{H}}\rangle_{0}=\frac{\langle\mathcal{H}\rangle_{0}}{L_{0} \theta_{0}^{2}}
$$

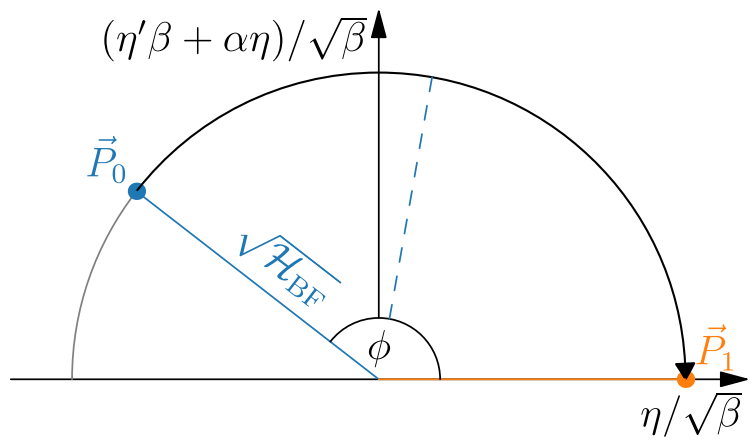

FIG. 2. Normalized phase space for dispersion. To enforce $\phi<\pi / 2, \vec{P}_{0}$ is rotated so that $\eta_{\vee}>0$ (dashed line).
Again using the drift-space approximation for $\beta(s)$ in the bending magnet and Eq. (2), this average can be rewritten as (Appendix B 1)

$$
\langle\hat{\mathcal{H}}\rangle_{0}=\frac{L_{0}}{\beta_{0}}\left[\left(\frac{\eta_{0}}{\theta_{0} L_{0}}\right)^{2}-\frac{1}{3}\left(\frac{\eta_{0}}{\theta_{0} L_{0}}\right)+\frac{1}{20}\right]+\frac{1}{3} \frac{\beta_{0}}{L_{0}} .
$$

\section{A. The TME condition}

Minimization of $\langle\hat{\mathcal{H}}\rangle_{0}$ with regard to $\beta_{0}, \eta_{0}$ yields the theoretical minimum emittance (TME) conditions [8]

$$
\frac{\beta_{0}^{\mathrm{TME}}}{L_{0}}=\frac{1}{\sqrt{15}} \approx 0.258, \quad \frac{\eta_{0}^{\mathrm{TME}}}{\theta_{0} L_{0}}=\frac{1}{6},
$$

and thus by Eq. (3) with the integral term simplifying to $\theta_{0} L_{0} / 2$ (see [7]), using Eqs. (7) and (12)

$$
\begin{aligned}
\left(I_{5} / I_{2}\right)_{\mathrm{TME}} & =\theta_{0}^{3} \frac{2}{3 \sqrt{15}}, \\
\phi_{\mathrm{TME}} & =\pi-\arctan \sqrt{\frac{3}{5} \approx 142.2^{\circ} .}
\end{aligned}
$$

The TME cell provides the minimum possible emittance for a single homogeneous bend per cell, but requires a large phase advance $2 \phi$. Also, considerable focusing into the bending magnet is required to reach the necessary $\beta_{0}$.

For the following parts of this work, all emittances are compared to that of the ideal TME cell (implying a homogeneous bending magnet). We therefore define the emittance ratio in accordance with [14] as

$$
F=\frac{I_{5} / I_{2}}{\left(I_{5} / I_{2}\right)_{\mathrm{TME}}} .
$$

\section{B. Emittance in the $\left(\phi, \beta_{0}\right)$ plane}

To obtain minimal emittances for a HOM cell at a fixed phase advance, one can insert the homogeneous case $b(s)=\theta_{0} / L_{0}$ into Eq. (3) and the phase relation following from Eq. (8), so that

$$
\eta_{0}\left(\beta_{0}, \phi\right)=\frac{1}{2} \theta_{0} L_{0}+\theta_{0} \beta_{0} \cot \phi .
$$

With this expression, Eq. (14) can be transformed to depend on $\beta_{0}$ and $\phi$,

$$
\begin{aligned}
& \langle\hat{\mathcal{H}}\rangle_{0}\left(\beta_{0}, \phi\right)=\frac{2}{15} \frac{L_{0}}{\beta_{0}}+A(\phi) \frac{\beta_{0}}{L_{0}}+\frac{2}{3} \cot \phi \\
& \text { with } \quad A(\phi)=\frac{1}{3}+\cot ^{2} \phi .
\end{aligned}
$$

The optics settings for minimal emittance at a given phase then follow via 


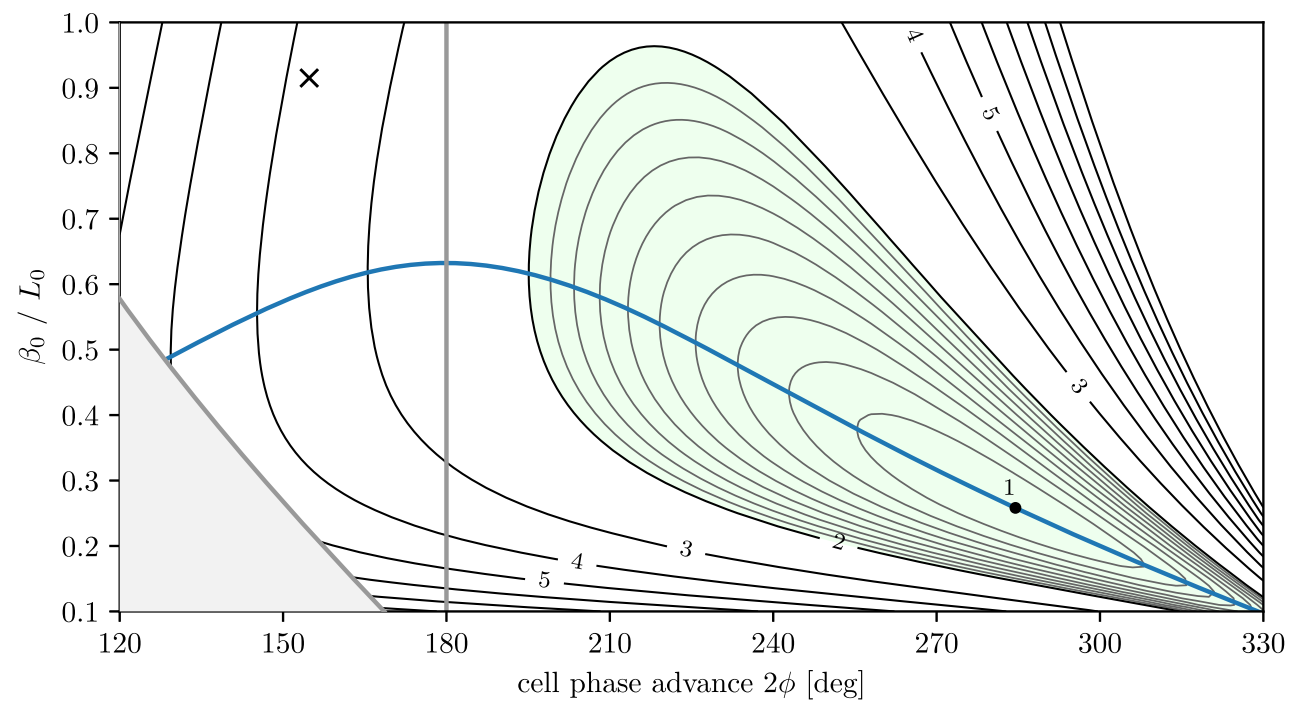

FIG. 3. Emittance ratio $F$ in the $\left(\beta_{0}, \phi\right)$ plane for HOM cells. Black isolines show values of $F$ in integer steps up to 10 . The gray isolines in the green area show values $F<2$ in steps of 0.1 . The blue line shows $\beta_{0}^{\text {opt }}(\phi)$, resulting in minimal emittance at a given phase. The TME condition $F=1$ is indicated by a black dot. The example cell parameters are indicated by the cross marker. Parameters in the gray area (bottom left) are not attainable.

$$
\frac{\beta_{0}^{\mathrm{opt}}(\phi)}{L_{0}}=\sqrt{\frac{2 / 15}{A(\phi)}} .
$$

The aforementioned relations for HOM cells are shown in Fig. 3. It is apparent that sensible phase advances $2 \phi<\pi$ can only be realized at significantly higher emittances $F>2.45$ relative to a cell fulfilling the TME condition.

\section{Homogeneous-bend (HOM) example cell}

In a series of examples throughout this work, we demonstrate the development of a lattice cell [15], starting with a HOM cell. All example cells have sufficient space to install additional sextupole magnets at proper positions, and their specifications can be found in Appendix A. The bend half-length was chosen as $L_{0}=0.2 \mathrm{~m}$, and assuming a beam energy of $2.4 \mathrm{GeV}$, the half angle was set to $\theta_{0}=2.5^{\circ}$. For these values, the emittance of a TME cell is $\varepsilon_{\mathrm{TME}}=121 \mathrm{pm}$.

A common technique to reduce the TME cell phase advance consists in detuning $\eta_{\mathrm{V}}$ to positive values so that $\phi<\pi / 2$ (see Fig. 2). The half-cell phase advances of the example cell were fixed at $\phi=0.43 \pi$ in the horizontal plane and $\phi_{\mathrm{y}}=0.13 \pi$ in the vertical plane-the higher horizontal than vertical tune results from focusing into the bend in order to achieve small emittance. Fixing the halfcell length to $1.1 \mathrm{~m}$ constrains $\beta_{0}$ to a larger-than-optimal value of $0.183 \mathrm{~m}$. The example cell parameters are also marked in the emittance surface in Fig. 3.

Figure 4 shows the optical functions and the magnetic field. The emittance of this HOM cell is $\varepsilon=454 \mathrm{pm}$ (or $F=3.75$ ).

\section{LONGITUDINAL GRADIENT BEND}

We continue the study by replacing the homogeneous bend with an LGB. As variation of field in a longitudinal gradient bend can be chosen arbitrarily, a general closedform solution without detailed specification of the field is at

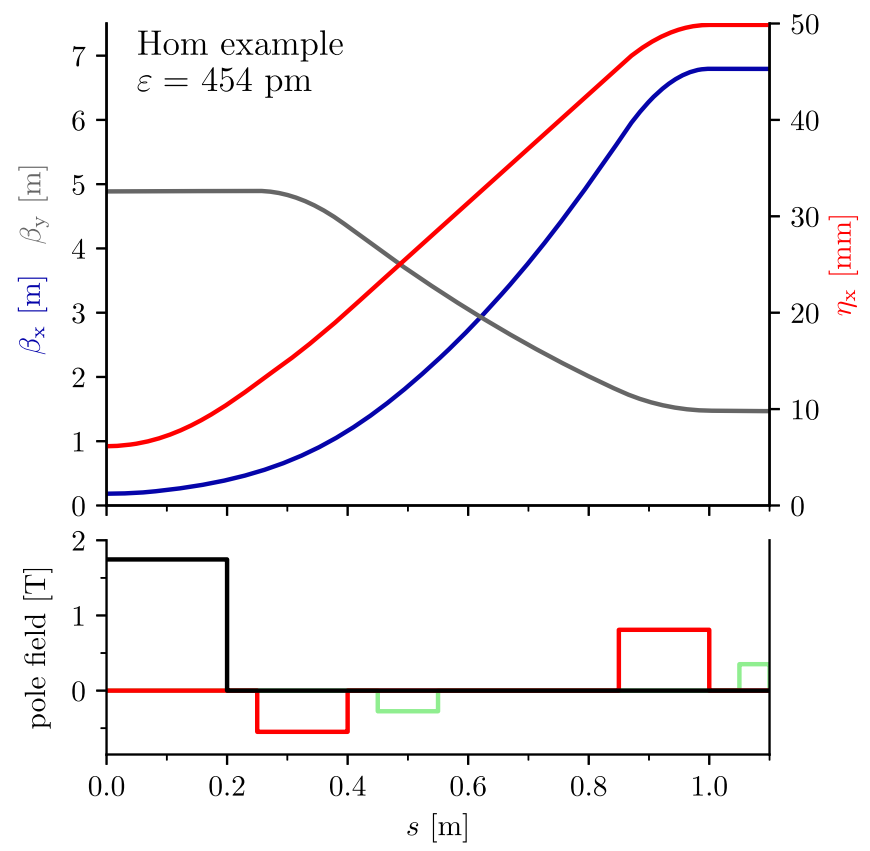

FIG. 4. Half cell with a homogeneous bend. The upper plot shows the optical functions $\beta_{\mathrm{x}}$ (blue), $\beta_{\mathrm{y}}$ (gray) and dispersion $\eta_{\mathrm{x}}$ (red). The lower plot shows the pole-tip field components for $R=13 \mathrm{~mm}$ half gap (or bore radius): dipole ( $B$, black), quadrupole $\left(B^{\prime} R\right.$, red), and sextupole $\left(B^{\prime \prime} R^{2} / 2\right.$, green). 
least cumbersome, and a variety of different magnet profiles for LGBs have been considered, e.g. [9-11].

In this work, we show elementary properties of LGBs using a simple curvature function with only one free parameter (Sec. IV B), and then generalize some properties to numerically optimized free-form LGBs (Sec. IV C).

Our description of the emittance contribution from longitudinal gradient bends closely follows [9]. To describe the variation of curvature in the bend, we define a normalized curvature function as

$$
\hat{b}(s)=\frac{L_{0}}{\theta_{0}} b(s), \quad \text { with } \quad\langle\hat{b}(s)\rangle=1,
$$

so that the emittance integrals in the bends can be expressed using averages via

$$
I_{5}^{(0)}=\frac{\left|\theta_{0}\right|^{5}}{L_{0}}\left\langle\left|\hat{b}^{3}\right| \hat{\mathcal{H}}\right\rangle_{0}, \quad I_{2}^{(0)}=\frac{\theta_{0}^{2}}{L_{0}}\left\langle\hat{b}^{2}\right\rangle_{0},
$$

and Eq. (9) simplifies to

$$
\frac{I_{5}}{I_{2}}=\theta_{0}^{3} \frac{\left\langle\left|\hat{b}^{3}\right| \hat{\mathcal{H}}\right\rangle_{0}}{\left\langle\hat{b}^{2}\right\rangle_{0}} .
$$

The numerator expression can be written as (Appendix B)

$$
\begin{aligned}
\left\langle\left|\hat{b}^{3}\right| \hat{\mathcal{H}}\right\rangle_{0} & =C \cdot \frac{\beta_{0}}{L_{0}}+\frac{L_{0}}{\beta_{0}} \cdot D\left(\frac{\eta_{0}}{\theta_{0} L_{0}}\right) \\
\text { with } C & =\left\langle\left|\hat{b}^{3}\right|\left(\frac{\eta^{\prime}}{\theta}\right)^{2}\right\rangle, \\
\qquad(x) & =\left\langle\left|\hat{b}^{3}\right|\right\rangle x^{2}-2\left\langle\left|\hat{b}^{3}\right| v\right\rangle x+\left\langle\left|\hat{b}^{3}\right| v^{2}\right\rangle .
\end{aligned}
$$

After division by the denominator term $\left\langle\hat{b}^{2}\right\rangle$, the magnetspecific coefficients of the $D(x)$ polynomial (defined using the lever function $v$, see Appendix B) and the coefficient $C$ replace constant values in the description of the homogeneous bend in Eq. (14). These parameters are dimensionless variants of the $\mathcal{I}_{n}$ terms for the symmetric bend in [9].

Based on Eq. (3), a further magnet-specific parameter $V$ is required to normalize the difference between the dispersion value $\eta_{0}$ at the center bend and the equivalent thin-dipole dispersion $\eta_{\vee}$ [7]

$$
\eta_{\vee}=\eta_{0}-V \theta_{0} L_{0} \quad \text { with } \quad V=\left\langle\hat{b} \frac{s}{L_{0}}\right\rangle .
$$

To characterize the concentration of magnetic field or curvature in the central bend region, we introduce the field enhancement factor [9]

$$
R=\frac{\max b(s)}{\langle b(s)\rangle}=\hat{b}(0)
$$

The four quantities $C, D, V, R$ fully describe the radiation and optics properties of the LGB in our model. The calculation of all required magnet-specific variables from the normalized curvature $\hat{b}(s)$ is shown in Appendix B.

\section{A. Emittance in the $\left(\phi, \beta_{0}\right)$ plane}

To obtain the emittance for a given phase advance of the cell, one inserts the phase relation following from Eqs. (25) and (8),

$$
\eta_{0}\left(\beta_{0}, \phi\right)=V \theta_{0} L_{0}+\theta_{0} \beta_{0} \cot \phi,
$$

into Eq. (24) so that

$$
\begin{aligned}
\left\langle\left|\hat{b}^{3}\right| \hat{\mathcal{H}}\right\rangle\left(\beta_{0}, \phi\right) & =\frac{L_{0}}{\beta_{0}} D(V)+A(\phi) \frac{\beta_{0}}{L_{0}}+\tilde{A} \cot \phi \\
\text { with } \quad A(\phi) & =C+\left\langle\left|\hat{b}^{3}\right|\right\rangle \cot ^{2} \phi \\
\tilde{A} & =2\left(\left\langle\left|\hat{b}^{3}\right|\right\rangle V-\left\langle\left|\hat{b}^{3}\right| v\right\rangle\right) .
\end{aligned}
$$

The calculation of optimal $\beta_{0}(\phi)$ is analogous to Eq. (20). Substituting this result into Eq. (28) yields

$$
\left\langle\left|\hat{b}^{3}\right| \hat{\mathcal{H}}\right\rangle^{\mathrm{opt}}(\phi)=2 \sqrt{D(V) A(\phi)}+\tilde{A} \cot \phi .
$$

The optimal phase can be derived from allowing the derivative with respect to $\cot \phi$ to vanish, so that

$$
2\left\langle\left|\hat{b}^{3}\right|\right\rangle \sqrt{D(V)} \cot \phi_{\mathrm{opt}}=-\tilde{A} \sqrt{A\left(\phi_{\mathrm{opt}}\right)} .
$$

The constraint $b(s) \geq 0$ results in $\tilde{A}>0$ (Appendix B). As all other quantities but $\cot \phi$ are also positive, this implies in our context that the optimal cell phase advance $2 \phi$ is larger than $\pi$ for any LGB cell with only positive curvature in its bend. In this case,

$$
\phi_{\mathrm{opt}}=\pi-\arctan \sqrt{\frac{4 D(V)\left\langle\hat{b}^{3} \mid\right\rangle^{2}}{C \tilde{A}^{2}}-\frac{\left\langle\left|\hat{b}^{3}\right|\right\rangle}{C}} .
$$

\section{B. Inverse distance-scaling magnet shape}

To proceed further, we need to specify the shape of longitudinal field variation $\hat{b}(s)$. We use the curvature function

$$
\hat{b}(s)=\frac{R}{w(s)} \quad \text { for } s \leq L_{0},
$$

where $w(s)=\sqrt{1+(s / h)^{2}}$ is a normalized distance to a point in the magnet midplane $s=0$ with a transverse offset $h$. Due to the inverse dependence of curvature on this 
distance, the shape is named "inverse distance-scaling magnet" (IDM) in the following. As a distinction between the usage of IDM and more general LGB field shapes as main bend, we refer to the former as "IDM cell" and to the latter as "LGB cell."

The IDM curvature has the advantage of being differentiable for all values $|s|<L_{0}$, leading to a smooth yoke shape (see, e.g., [23]). For $s>L_{0}$, the curvature vanishes. It thus can in principle be realized "as-is" when not considering fringe fields at the magnet end.

The field enhancement factor for the IDM shape is then given by

$$
R=\frac{L_{0} / h}{\operatorname{arsinh}\left(L_{0} / h\right)}
$$

In the limit $h \rightarrow \infty$, equivalent to $R \rightarrow 1$, the IDM reduces to a homogeneous magnet of length $L_{0}$ (Sec. III A). Emittance integrals and related quantities are computed in Appendix B.

The properties of IDM cells in dependence of $R$ are shown in Fig. 5. For optimized cell phase advance $\phi$ and $\beta_{0}$, the emittance of the LGB cell relative to a HOM cell with equal $\phi, \beta_{0}$ can be significantly reduced for increasing $R$. This optimal phase advance unfortunately increases with $R$, such that for any given phase in the region of interest $(2 \phi<\pi)$, there exists a critical field enhancement factor above which the IDM cell emittance is actually larger than that of a HOM cell. We can also observe that in the region of interest, $F<2$ is not possible. On the other hand, we observe that $R \leq 2$ is sufficient. This missing emittance reduction is a result of the improperly matched optics at the bend for sensible phase advances (especially the lower dispersion bound $\eta_{0}>V \theta_{0} L_{0}$ for $\left.2 \phi<\pi\right)$.

To select a proper field enhancement for a study of the $\left(\phi, \beta_{0}\right)$ plane, we choose $R=2$ which is moderate and technically feasible for many setups and in principle allows significant emittance reduction. The characteristics of the LGB cell with this field enhancement factor are shown in Fig. 5 (bottom left). On the one hand, the capability of the IDM cell in reducing emittance for large phase advances relative to HOM cells is again obvious. On the other hand, the emittance reduction for sensible phase advances is marginal, although having the advantage of being robust toward increasing $\beta_{0}$.
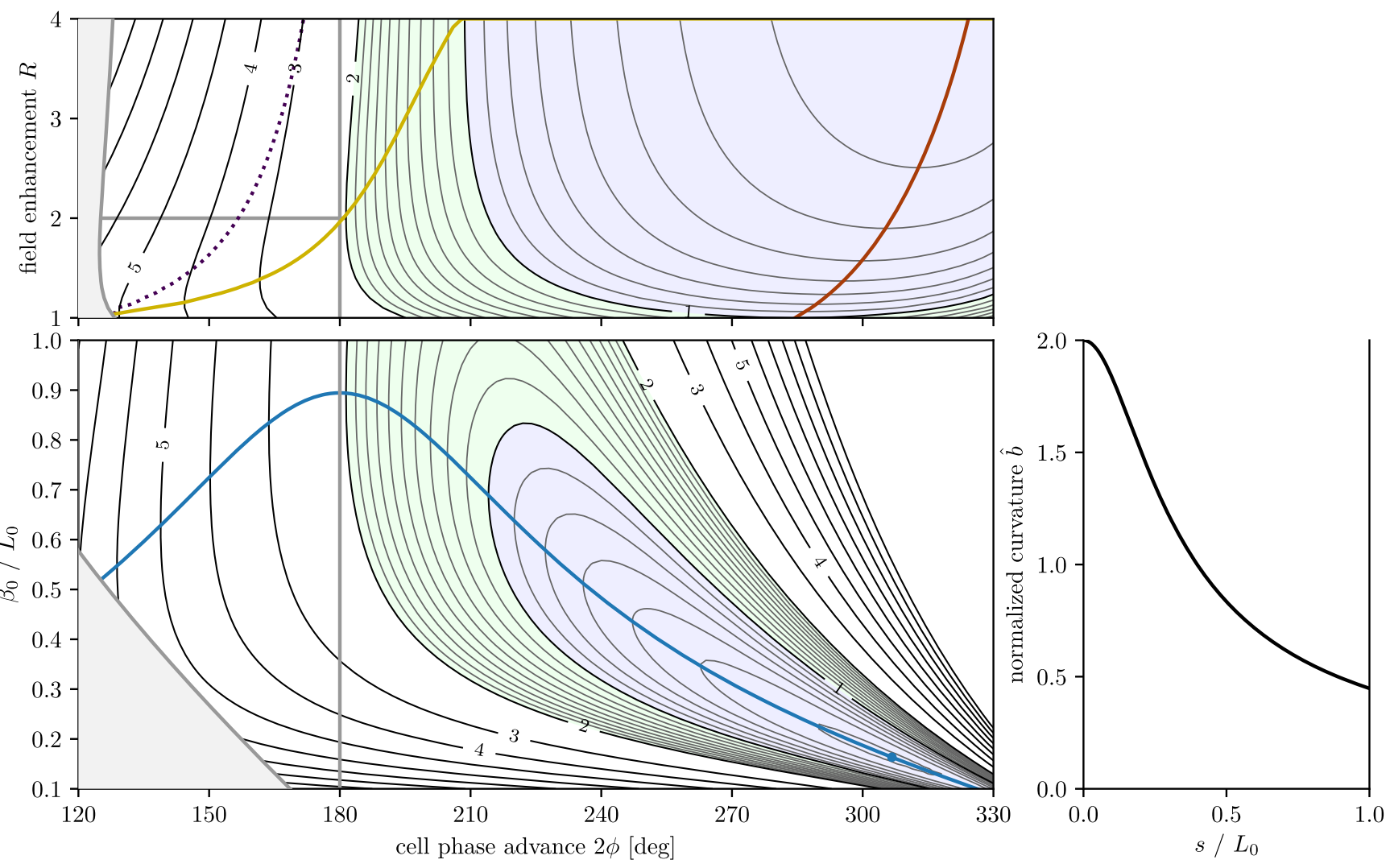

FIG. 5. Properties of the IDM shape. Top: TME emittance ratio $F$ for optimal $\beta_{0}$ in dependence of $R$, $\phi$. The red line shows $\phi_{\text {opt }}$ for given $R$. The dark-yellow line shows the optimal field enhancement $R$ for given $\phi$. Above the dotted line (critical field enhancement), a HOM cell tuned to the LGB-optimal parameters $\phi, \beta_{0}^{\text {opt }}$ yields lower emittance than the LGB cell. The region with $F<1$ is shaded in blue (see legend in Fig. 3). Bottom left: $F$ in the $\left(\beta_{0}, \phi\right)$ plane for an IDM with $R=2$; the optimum emittance is denoted with a blue dot. Right: IDM on-axis field profile for $R=2$. 

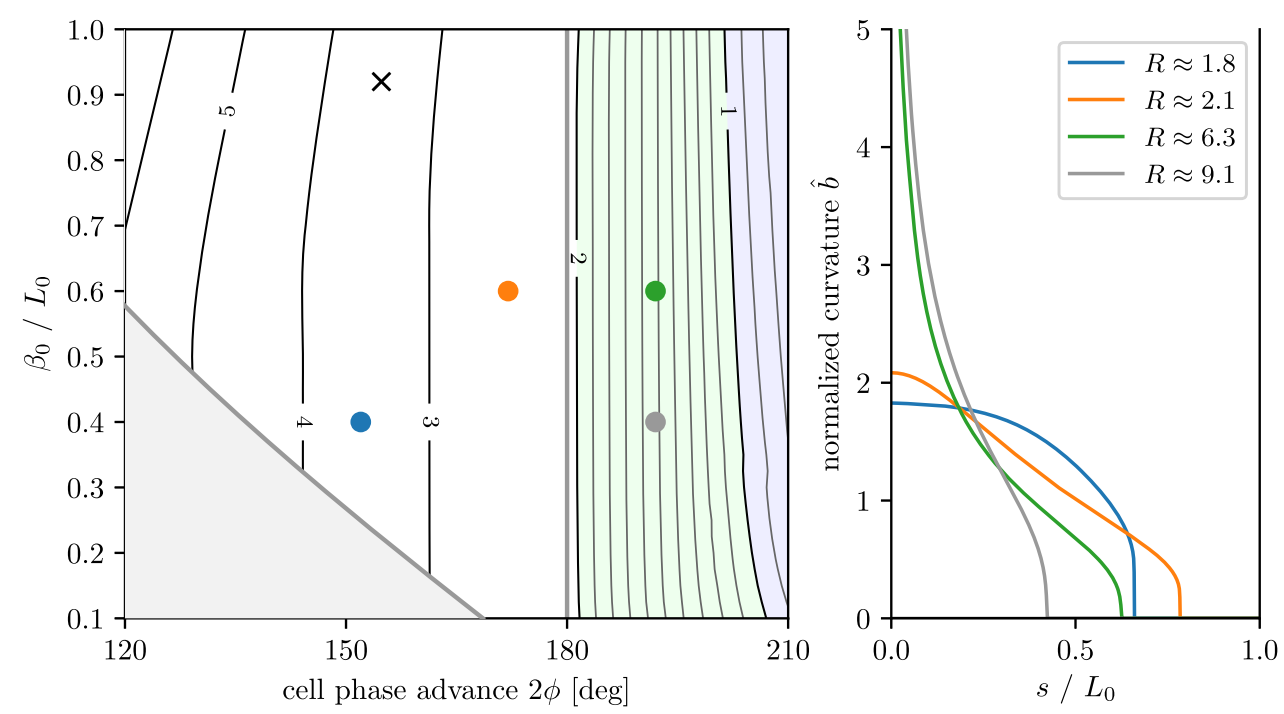

FIG. 6. TME emittance ratio $F$ and example curvatures for free-form LGB cells with the constraint of positive curvature $b(s) \geq 0$. Left: $F$ in dependence of $\beta_{0}, \phi$ (see legend in Fig. 5). Colored markers denote the curvature examples in the right plot. Right: curvature examples for setups marked in left plot.

\section{Optimized free-form LGBs with positive curvature}

Is the limited performance of IDM cells for sensible phase advances a phenomenon that generalizes to all LGB cells? For given $\phi, \beta_{0}$, we strive to find a curvature shape with $b(s) \geq 0$ that minimizes $F$, in other words the functional $F(b)$. While dipole field optimizations have been performed in many other works, the transfer matrix of the cell is either not kept constant during optimization [11,24], resulting in detuning of $\beta_{0}, \phi$ during the procedure; or the projected dispersion and its derivative are set to vanish at some position in the lattice [25].

Since there is an infinite space of possible field shapes $b(s)$, we need to apply reasonable assumptions for the following search. For the field shape to be a physical solution, it should be possible to create it as a perturbation of a homogeneous bend, and it should thus be accessible by local optimization, using the parameters of a homogeneous bend as initial values.

The optimal shape can be approximated numerically by discretising $\hat{b}(s)$ into values $\underline{b}_{q}$ (details in Appendix B 3). We apply automatic differentiation [26,27] to obtain the gradient of the objective function $F\left(\underline{b}_{1}, \ldots \underline{b}_{Q}\right)$. The objective function and its gradient are used as input to the limited-memory Broyden-Fletcher-Goldfarb-Shanno algorithm with box constraints (L-BFGS-B) [28].

The optimization is carried out independently for each point on a grid in the $\left(\phi, \beta_{0}\right)$ plane. For all points with the largest value of $\beta_{0} / L_{0}=1$, the initial values are set to $\underline{b}_{q}=1$, equivalent to a homogeneous bend. For all other points, the optimized shape from the next-larger $\beta_{0} / L_{0}$ value at equal $\phi$ is used for initialization, requiring the optimization loop along the $\beta_{0}$ dimension to be carried out in reverse order. To prevent the numerical discretization of $b(s)$ from influencing convergence at very high field enhancement factors, we limit the scope of our optimization study to $2 \phi \leq 210^{\circ}$.

The results of this computation are shown in Figs. 6 and 7. Emittance in the $\left(\phi, \beta_{0}\right)$ plane is always reduced relative to the TME case (Fig. 3), and the achieved emittance is robust regarding changes in $\beta_{0}$ : due to the length-related quantities $\beta, \eta$ in our model scaling with $L_{0}$, one can always find a similar $F$ value when reducing $\beta_{0}$ and the $s$ dimension of $\hat{b}(s)$ simultaneously while keeping its integral constant ("squeezing"). The optimization shows this effect if lower values of $F$ cannot be found by reducing $\beta_{0}$ and leads to reduced magnet lengths (see example shapes in Fig. 6).

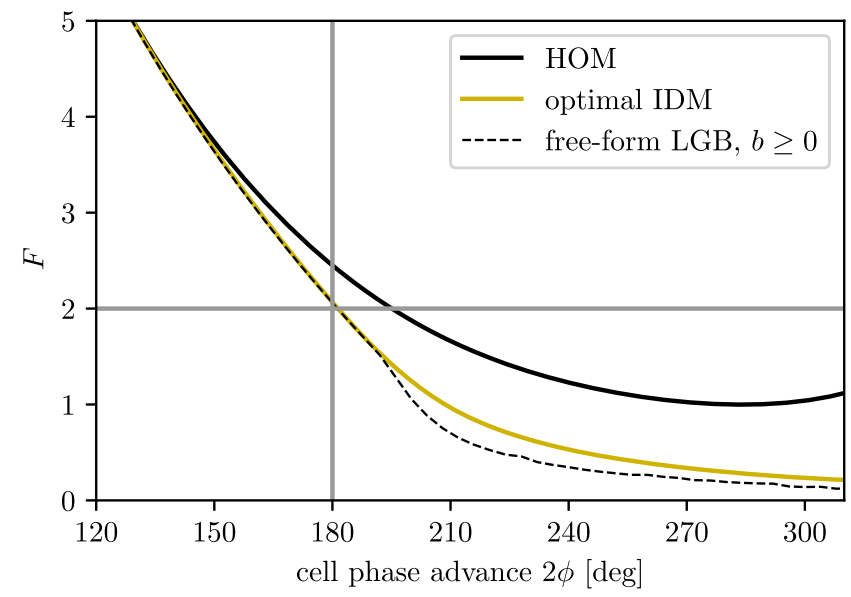

FIG. 7. Comparison of minimum possible emittances for HOM cells, optimized LGB cells with positive curvature, and IDM cells. 


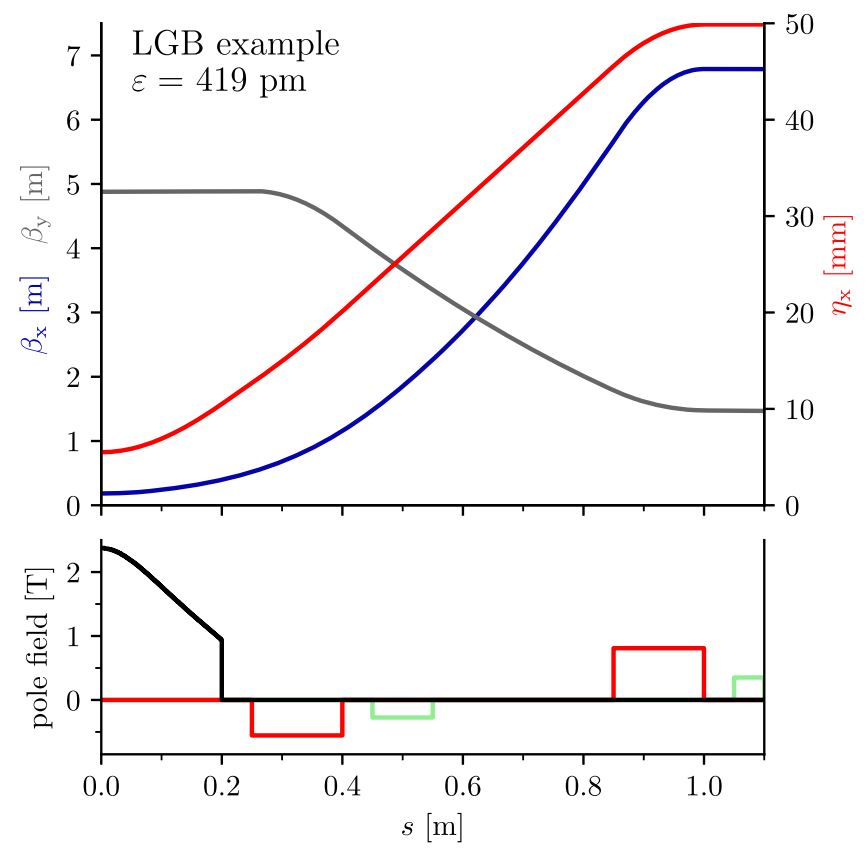

FIG. 8. Example half cell with optimization of longitudinal field variation $(b \geq 0)$ in its bend (see Fig. 4 for legend.)

On the one hand, significant emittance reduction is possible for large phase advances, where high field enhancement factors occur for the magnet shapes (like for the IDM special case). On the other hand, within our assumptions we observe that for sensible phase advances $2 \phi<\pi$, no optimized shape leads to a TME emittance ratio $F \leq 2$. In this regime, IDM shapes with $R \leq 2$ at their optimal $\beta_{0}$ values actually yield a comparable performance to the optimized LGB shapes (Fig. 7).

\section{LGB example cell}

As a continuation of the example of Sec. III C, a longitudinal gradient bend is introduced by insertion of the appropriate optimized field profile for given $\beta_{0}$ and $\phi$ as obtained in the last section. After insertion of the modified bend, slight modifications of quadrupole strengths are required to maintain $\phi, \phi_{\mathrm{y}}$ at their previous values (with almost equal $\beta_{0}=0.184 \mathrm{~m}$, example parameters marked in Fig. 6). The cell optics and magnet characteristics are shown in Fig. 8. The LGB field possesses a moderate enhancement factor of $R \approx 1.4$ and leads to an emittance $\varepsilon=419 \mathrm{pm}$ (or $F=3.46$ ) of this example LGB cell, which is a marginal improvement by $7.7 \%$ relative to the HOM example (this reduction is mainly produced by an increase of $I_{2}$ rather than reduction of $I_{5}$, see Fig. 18). Both findings are consistent with our model predictions regarding sensible phase advances $2 \phi<\pi$.

\section{E. Optimized free-form LGBs with arbitrary curvature}

Constraining the bend to possess only positive curvatures $b(s) \geq 0$ and sensible phase advances $2 \phi<\pi$ causes $\eta_{0}>V \theta_{0} L_{0}>0$ to have a finite, positive lower bound. In consequence, the dispersion action $\mathcal{H}(s)$ in the bending magnet also has a lower bound-enhancing the peak field of an LGB can only minimize $I_{5}$ and thus the emittance to a given extent. To investigate this limit in the following, the constraint $b(s) \geq 0$, which has been applied in the previous optimization of the free-form LGB shapes, is removed.

The full optimization procedure is again performed on the curvature shapes using L-BFGS-B [28], but without application of bounds on $\underline{b}_{q}$. We obtain significantly lower emittances in the region $2 \phi<\pi$ compared to the case of positive curvatures (compare Fig. 9 with Fig. 6)—indeed
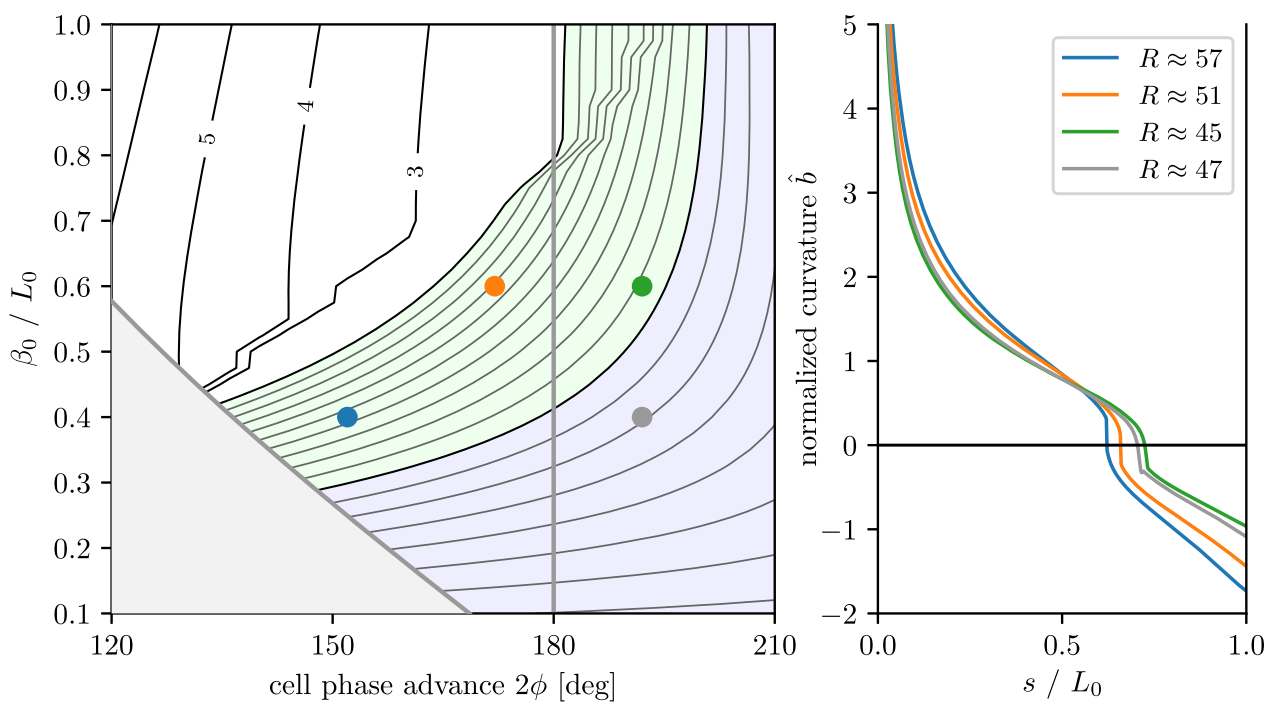

FIG. 9. TME emittance ratio $F$ and example curvatures for optimized LGB cells without sign constraints on $b(s)$ (see Fig. 6 for legend). 
emittances $F<2$ and even $F<1$ are possible, although large field enhancement is required.

The interesting regions in Fig. 9 (left), where the phase advance is sensible $(2 \phi<\pi)$ and small emittance $(F \sim 1)$ is obtained, are adjacent to the region where all phase advance is contained inside the bend [gray shaded area defined by Eq. (1)]. This means that the bend basically fills the cell. As visible in Fig. 9 (right), the curvature switches sign-one may interpret this behavior as the free-form LGB actually splitting into a main bend of positive curvature and a reverse bend $(\mathrm{RB})$ of negative curvature. There is no focusing element located between the bends, so that $\beta(s)$ behaves like in a drift space and is only refocused at the cell end. The focusing element could be imagined as a thin quadrupole as studied in [14]: in this idealized setup, $\beta(s)$ also behaves like in a drift space over the full cell length, containing bends of opposite polarity.

The significant reduction at sensible phase advance is due to a combined action of LGB and RB. With the RB at the end of the cell, the phase advance between RB and main bend is not much less than $90^{\circ}$, so a negative kick on dispersion $-\Delta \eta_{1}^{\prime}$ applied by the RB translates in a reduction of $\eta_{0}$, and with it $\mathcal{H}_{0}$, at the main bend center, which enables the LGB to efficiently suppress emittance by adjusting the curvature $b(s)$ appropriately.

Unboundedness of $b(s)$ means that $V$ and thus $\eta_{0}$ can be chosen more or less freely for a given transfer matrix by adjusting the RB acting as lever at the cell end (Fig. 10). We also note from the optimized shapes in Fig. 9 that the reverse-bend strength is largest near the magnet end, where $\beta(s) \propto s^{2}$ reaches its maximum. This is reasonable as perturbations in orbits and dispersion scale with $\sqrt{\beta}$, making adjustments there more effective.

At this point, we refer to the recent work [29], in which a general numerical optimization of a periodic lattice cell is performed using the different approach of parametrization

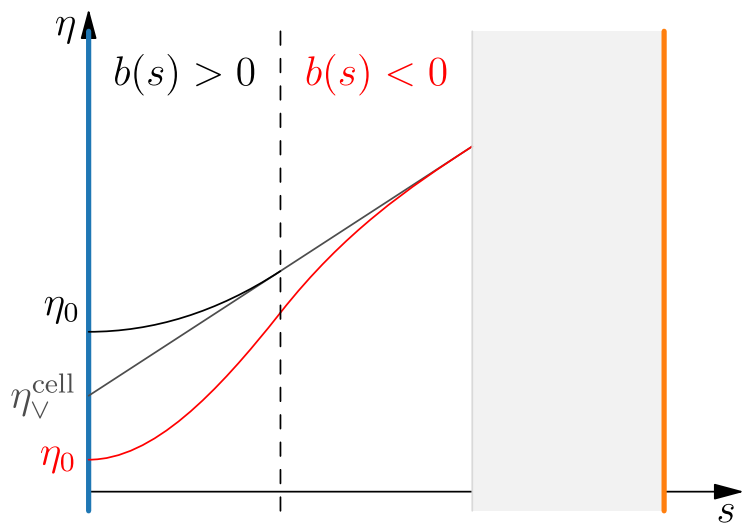

FIG. 10. Behavior of dispersion in an LGB cell for constant $\beta_{0}$ and $\phi<\pi / 2$ (and thus $\eta_{\vee}>0$ ) with only positive curvature (black) and with alternating curvature (red). The reverse curvature allows $\eta_{0}<\eta_{\vee}$. To keep the total bending angle constant including negative curvature, the overall positive curvature is increased. and optimization of distributed magnet strengths. Most interestingly, using an emittance minimization objective, the resulting dipole field shapes in [29] are qualitatively similar to the $b(s)$ shapes in Fig. 9. This increases our confidence that reverse bending is an intrinsic feature of low-emittance lattice design.

\section{REVERSE-BEND CELLS}

Since reverse bends naturally emerge from the optimization of the free-form LGB and reveal the potential to realize $F<2$ emittance at sensible phase advance $(2 \phi<\pi)$, we proceed with a study of a modified unit half-cell composed from two discrete magnets, which may possess opposite polarities. Unit cells including reverse bends have been considered for a wiggler storage ring [12], are an established concept used for damping rings [13] and have recently been suggested for use in modern synchrotron light sources [14].

A generalization of the dispersion matching condition from Sec. II to cells with two bends allows to keep the following study free from the specification of focusing elements. A second bending magnet with full length $2 L_{1}$ and a total bending angle $2 \theta_{1} \neq 0$ is introduced at the opposite half-cell end (see Fig. 11). We define the equivalent thin-kick dispersion for the second bend in analogy to Eq. (3) as

$$
\eta_{\wedge}=\eta_{1}-\int_{0}^{L_{1}} s \cdot b(s) \mathrm{d} s .
$$

Then the condition for dispersion matching Eq. (5) modifies to

$$
\mathbf{B}_{1}^{-1}\left(\begin{array}{c}
\eta_{\wedge} \\
-\theta_{1}
\end{array}\right)=\mathbf{R}(\phi) \mathbf{B}_{0}^{-1}\left(\begin{array}{c}
\eta_{\vee} \\
\theta_{0}
\end{array}\right)
$$

so that Eq. (6) holds with a more general expression for

$$
\vec{P}_{1}=\frac{1}{\sqrt{\beta_{1}}}\left(\begin{array}{c}
\eta_{\wedge} \\
-\theta_{1} \beta_{1}
\end{array}\right)
$$

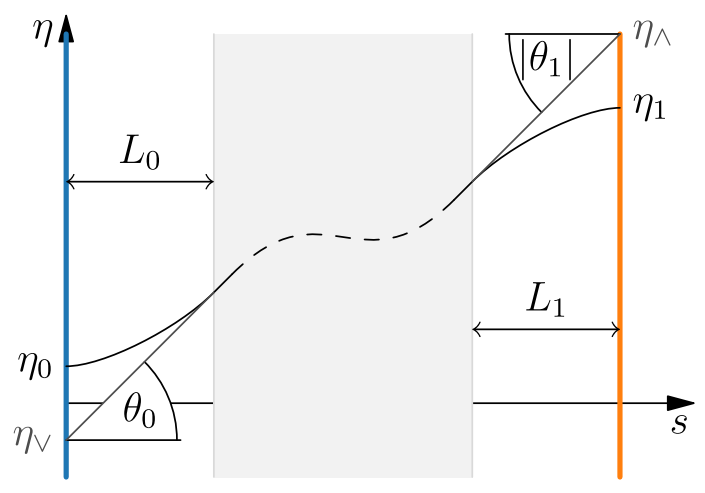

FIG. 11. Dispersion $\eta_{0}, \eta_{1}$ and equivalent thin-dipole dispersion $\eta_{\vee}, \eta_{\wedge}$ for a half cell with unspecified interior and $\theta_{1}<0$ (compare to Fig. 1). 


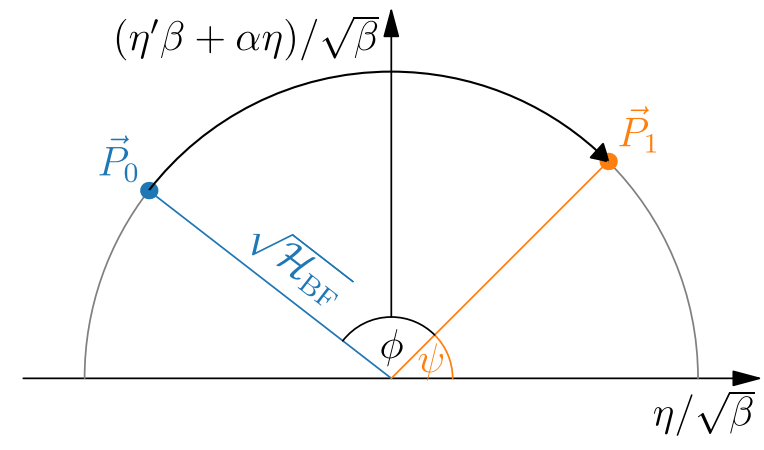

FIG. 12. Normalized phase space for dispersion in a two-bend cell with $\theta_{0}>0, \theta_{1}<0$ (reverse-bend cell).

The dispersion matching in this general cell is sketched in Fig. 12 with the additional definition

$$
\psi=\operatorname{atan} 2\left(-\theta_{1} \beta_{1}, \eta_{\wedge}\right)
$$

so that when denoting the cell phase advance from Eq. (7) as $\phi_{\text {iso }}$, we obtain modified phase advance and dispersion expressions

$$
\begin{gathered}
\phi=\phi_{\text {iso }}-\psi, \quad \eta_{\vee}=\theta_{0} \beta_{0} \cot \phi_{\text {iso }}, \\
\theta_{1} \sqrt{\beta_{1}}=-\sqrt{\mathcal{H}_{\mathrm{BF}}} \sin \psi, \quad \eta_{\wedge}=-\theta_{1} \beta_{1} \cot \psi .
\end{gathered}
$$

The phase advance of a two-bend cell is reduced relative to an single-bend cell with identical $\beta_{0}, \eta_{\vee}$ values only if

$$
\psi \in] 0, \pi\left[\Leftrightarrow-\theta_{1} \beta_{1}>0 \Leftrightarrow \theta_{1}<0\right.
$$

so that the additional dipole magnet bends the beam in opposite direction relative to the main bend. This setup is known as a reverse-bend cell (or antibend cell) [14].

As transverse kicks scale with $\sqrt{\beta}$, we expect a relation between $\beta_{1}$ and reverse bend angle $\left|\theta_{1}\right|$. For larger $\beta_{1}$, the reverse bend will become more effective, requiring less bending angle and thus contributing less to the emittance, which naturally makes optics settings with large $\beta_{1}$ beneficial for reverse-bend cells. In general, however, the parameter $\beta_{1}$ is limited towards large values by optics constraints (e.g., cell length, see also Sec. VI), which need to be balanced with the available emittance reduction.

Furthermore, we mention that the linear momentum compaction, proportional to the radiation integral

$$
I_{1}=\int \eta(s) b(s) \mathrm{d} s
$$

is reduced for reverse-bend cells relative to cells with $b(s)>0$. This quantity should be considered in a realistic lattice design and also depends on the particular field shapes in each bend.

\section{A. Relative length of bending magnets}

As $\eta(s)$ and $\beta(s)$ in the $\mathrm{RB}$ region are rather large and do not vary much, the gain from a longitudinal field variation would be negligible, and one can therefore assume the RB to be homogeneous. In consequence, its radiation properties are fully characterized by Eq. (14) when replacing the index 0 with 1 ,

$$
\left\langle\left|\hat{b}^{3}\right| \hat{\mathcal{H}}\right\rangle_{1}=\langle\hat{\mathcal{H}}\rangle_{1} \quad \text { with } \quad \eta_{1}=\eta_{\wedge}+\frac{1}{2} \theta_{1} L_{1}
$$

Then the ratio of radiation integrals depends on the ratio of bending magnet lengths,

$$
\begin{aligned}
\frac{I_{5}^{(0)}+I_{5}^{(1)}}{I_{2}^{(0)}+I_{2}^{(1)}} & =\theta_{0}^{3} \frac{\left\langle\left|\hat{b}^{3}\right| \hat{\mathcal{H}}\right\rangle_{0}+\left(L_{0} / L_{1}\right)|\hat{\theta}|^{5}\langle\hat{\mathcal{H}}\rangle_{1}}{\left\langle\hat{b}^{2}\right\rangle_{0}+\left(L_{0} / L_{1}\right) \hat{\theta}^{2}}, \\
\text { with } \hat{\theta} & =\theta_{1} / \theta_{0} .
\end{aligned}
$$

For simplicity we will assume that the RB has approximately same field strength like a homogeneous main bend, $b=\theta_{0} / L_{0}$, although eventually its length $L_{1}$ may be optimized with regard to minimum contribution to $I_{5}$ (long RB) on one side and minimum cell length (short RB) on the other side. Then,

$$
L_{1} / L_{0}=|\hat{\theta}|
$$

To compare emittances of unit cells with two bends with that of the TME cell, their half-cell bending angles should be equal. Defining this angle as $\Theta=\theta_{0}+\theta_{1}$ for unit cells with two bends, we use the relation $\Theta / \theta_{0}=1+\hat{\theta}$ to obtain the TME emittance ratio of a general unit cell with bend lengths defined by Eq. (44) as

$$
\begin{aligned}
F= & \frac{3 \sqrt{15}}{2} \frac{\left\langle\left|\hat{b}^{3}\right| \hat{\mathcal{H}}\right\rangle_{0}+\hat{\theta}^{4}\langle\hat{\mathcal{H}}\rangle_{1}}{(1+\hat{\theta})^{3}\left(\left\langle\hat{b}^{2}\right\rangle_{0}+|\hat{\theta}|\right)}, \text { with } \\
\hat{\theta}^{4}\langle\hat{\mathcal{H}}\rangle_{1}= & \frac{L_{0}}{\beta_{1}}\left[|\hat{\theta}|\left(\frac{\eta_{1}}{\theta_{0} L_{0}}\right)^{2}-\frac{\hat{\theta}^{3}}{3}\left(\frac{\eta_{1}}{\theta_{0} L_{0}}\right)+\frac{\left|\hat{\theta}^{5}\right|}{20}\right] \\
& +\frac{\left|\hat{\theta}^{3}\right|}{3} \frac{\beta_{1}}{L_{0}} .
\end{aligned}
$$

\section{B. Degrees of freedom and optimization}

Besides the characteristics of the main bending magnet, the emittance ratio $F$ of the reverse-bend cell in Eq. (45) is fully defined by $\beta_{0}, \eta_{0}$ at the main-bend center, $\beta_{1}, \eta_{1}$ at the reverse-bend center (all lengths and angles in units of $L_{0}$, $\theta_{0}$ ), and $\hat{\theta}$. These five parameters are also sufficient to find the half-cell phase advance $\phi$. One degree of freedom is absorbed by enforcing $\vec{P}_{0}$ and $\vec{P}_{1}$ to be located on a circle (equal $\mathcal{H}_{\mathrm{BF}}$, Fig. 12), so that four degrees of freedom remain. 
To obtain some insight into the emittance properties of the cell relative to cells with one bend, we choose the free parameters as $\left(\beta_{0}, \phi, \beta_{1}, \hat{\theta}\right)$. One may then numerically find the optimal $F$ for a given set of parameters $\left(\beta_{0}, \phi, \beta_{1}\right)$ with the free parameter $\hat{\theta}$ using, e.g., direct search as performed in the following cases, and show slices of the resulting three-dimensional parameter space.

\section{Reverse-bend cells with homogeneous main bend (HOM/RB cells)}

For a homogeneous main bend, one can replace $\left\langle\left|\hat{b}^{3}\right| \hat{\mathcal{H}}\right\rangle_{0}$ in Eq. (45) with $\langle\hat{\mathcal{H}}\rangle_{0}$ from Eq. (14) and set $\left\langle\hat{b}^{2}\right\rangle_{0}=1$, obtaining a simplified emittance expression

$$
F=\frac{3 \sqrt{15}}{2} \frac{\langle\hat{\mathcal{H}}\rangle_{0}+\hat{\theta}^{4}\langle\hat{\mathcal{H}}\rangle_{1}}{(1+\hat{\theta})^{3}(1+|\hat{\theta}|)} .
$$

Although $\langle\hat{\mathcal{H}}\rangle_{1}>\langle\hat{\mathcal{H}}\rangle_{0}$, the $\hat{\theta}^{4}\langle\hat{\mathcal{H}}\rangle_{1}$ summand is small relative to $\langle\hat{\mathcal{H}}\rangle_{0}$. The major effect on the emittance is then given by $\langle\hat{\mathcal{H}}\rangle_{0}$, as in the TME cell, and the changes in bending angle described by the denominator. Thus we can expect that without constraints, the optimal values of $\beta_{0}, \eta_{0}$ will only slightly deviate from those of the TME cell.

The results of the numerical optimization for given $\beta_{0}, \phi_{0}, \beta_{1}$ are presented in Fig. 13. The parameter space splits into three regions with regard to $\theta_{1}$. A small region with vanishing $\theta_{1}$ exists that is limited toward small and large phase advances; the emittance in this region is thus identical to that of a HOM cell. For large phase advances, $\theta_{1}>0$, and the emittance reduces even below $F<1$. This behavior can be interpreted as the two-bend cell approximating a double-period TME cell, which would be reached at $\phi=2 \phi_{\mathrm{TME}}, \beta_{0}=\beta_{0}^{\mathrm{TME}}$ and would result in $F=1 / 8$.

For sensible phase advances $2 \phi<\pi$, the bending angle of the second bend is reversed as expected. Comparing to the considered one-bend cells with positive curvature, the $F<2$ region now extends into the range of sensible phase advance, so that lower emittances are feasible.

One can furthermore observe the found relation between $\beta_{1}, \theta_{1}$, and emittance: low $\left|\theta_{1}\right|$ allows to reach lower emittances, but requires large $\beta_{1}$ values, while larger absolute reverse-bend angles allow moderate $\beta_{1}$ values at less emittance reduction.

We conclude that at moderate reverse bending angles $\left|\theta_{1}\right|<\theta_{0} / 5$, significantly lower emittances than with an LGB or HOM cell are possible for the interesting range of phase advances due to prevention of optical mismatch at the main bend, which is a clear advantage of this cell type.

\section{HOM/RB example cell}

Adding a small reverse bend of length $L_{1}=0.05 \mathrm{~m}$ ( $\left.L_{1}=L_{0} / 4\right)$ to the HOM example cell (Sec. III C) increases its length, but effectively reduces the dispersion at the main bend center, see Fig. 14. The reverse bend is shifted from the half-cell end, allowing for the installation of a sextupole magnet at that symmetry point and leading to half-cell length of $1.2 \mathrm{~m}$ (Appendix A).

Here the RB angle was set to $\theta_{1}=-0.2^{\circ}$, and the angle of the main bend $\theta_{0}$ was increased by $-\theta_{1}$ to maintain the total cell deflection $(\hat{\theta}=0.074)$. As a consequence the field in the main bend is higher since its length was maintained. At unchanged phase advances $\phi, \phi_{\mathrm{y}}$ (resulting in increased
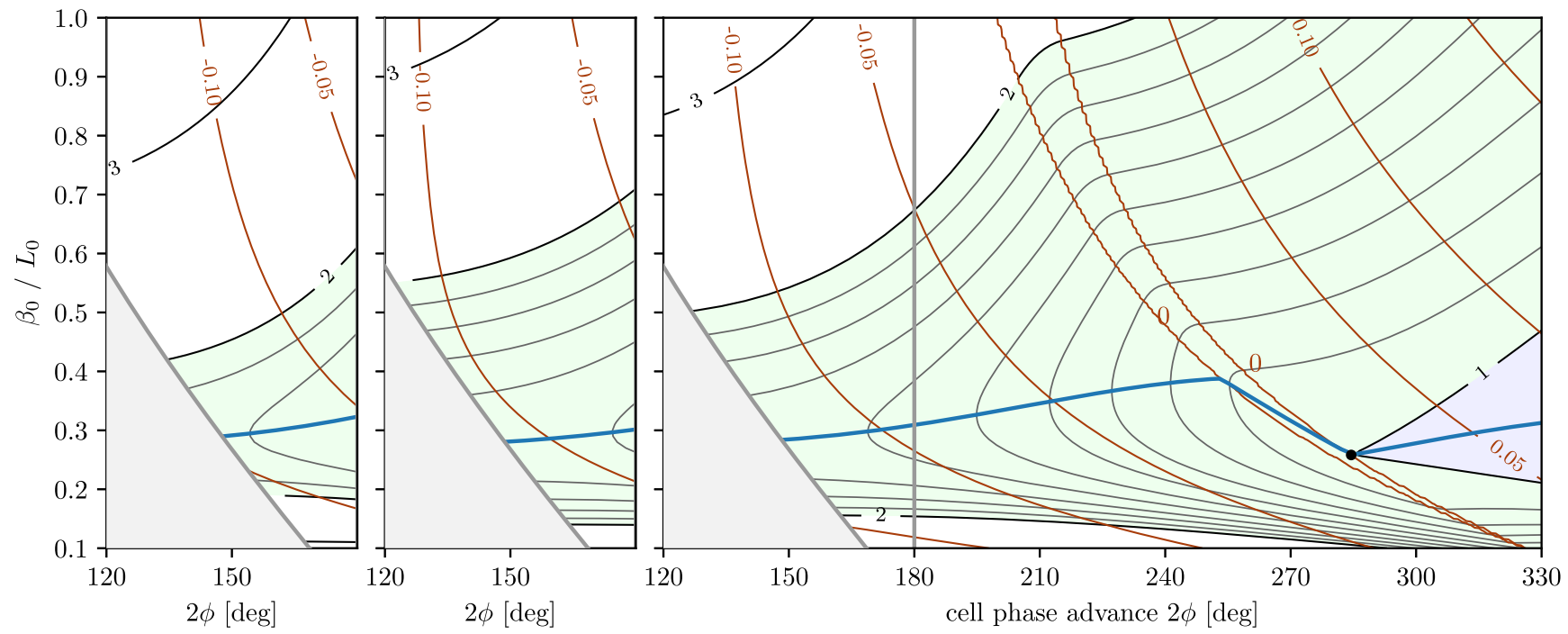

FIG. 13. Emittance ratio $F$ for RB cells (see legend in Fig. 3) with different values of $\beta_{1}$ at the reverse-bend center. From left to right: $\beta_{1}=20 L_{0}, \beta_{1}=40 L_{0}, \beta_{1}=30 L_{0}$. Red lines show $\theta_{1} / \theta_{0}$ for the respective cells in steps of 0.05 . At large phase advances, two isolines for $\theta_{1}=0$ (actually $\pm 10^{-4}$ ) delimitate a "plateau" of vanishing reverse bending angle, the black dot marking the TME condition like in Fig. 3. 


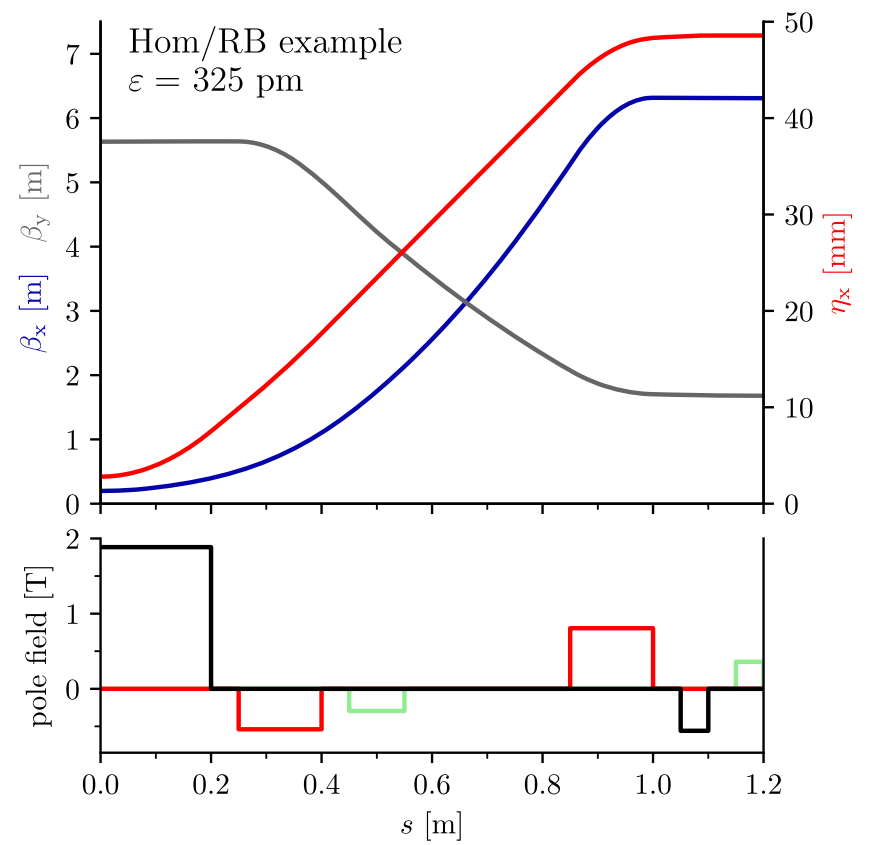

FIG. 14. Example RB cell with homogeneous main bend, utilizing a reverse bend for suppression of dispersion at main bend (see Fig. 4 for legend.)

$\beta_{0}=0.198 \mathrm{~m}$ ), the emittance shrinks to $\varepsilon=325 \mathrm{pm}$ (or $F=2.69$ ), which is a reduction of $28 \%$ relative to the HOM cell example (Sec. III C).

\section{E. Reverse-bend cells with IDM as main bend (IDM/RB cells)}

Lowering $\eta_{0}$ by means of RBs eventually enables an LGB as main bend to efficiently reduce the emittance: a central peak of high field concentrates most bending and quantum emission in a region of small $\mathcal{H}$, while the decay of field strength towards the edges compensates for the inevitable growth of $\mathcal{H}$, thus minimizing the radiation integral $I_{5}$.

When optimizing the LGB, two concerns have to be taken into account: As the width of a magnetic field peak cannot be significantly smaller than the magnet gap, such a large field enhancement $R$ does not correspond to a realistic magnet design. Furthermore, the equilibrium energy distribution of particles [22],

$$
\sigma_{\delta}^{2} \propto \frac{I_{3}}{I_{2}} \quad \text { with } \quad I_{3}=\int\left|b^{3}(s)\right| \mathrm{d} s,
$$

may become too large for high peak field, as $I_{3} / I_{2}$ generally increases with $R$. Thus, a realistic model to be investigated is a unit cell using an IDM with limited $R$ as main bend, and a homogeneous reverse bend.

The necessary computations have already been carried out in Sec. IV, resulting in Eq. (24), Eq. (25), and at the beginning of this section, resulting in Eq. (45). We again use an IDM with field enhancement factor $R=2$ and insert its parameters into the numerical optimization procedure for RB cells.

The results of this procedure are shown in Fig. 15. Like for HOM/RB cells, the region of low emittances is extended into the range of phase advances $2 \phi<\pi$ by virtue of the reverse-bend scheme.

As can be observed in comparison with Fig. 13, the emittance of an IDM/RB cell can be significantly smaller than that of a HOM/RB cell and potentially even reach $F \leq 1$.

We conclude that the figures of merit being low cell phase advance, optics matching in the main bend and exploitation of LGB characteristics cannot not be reached
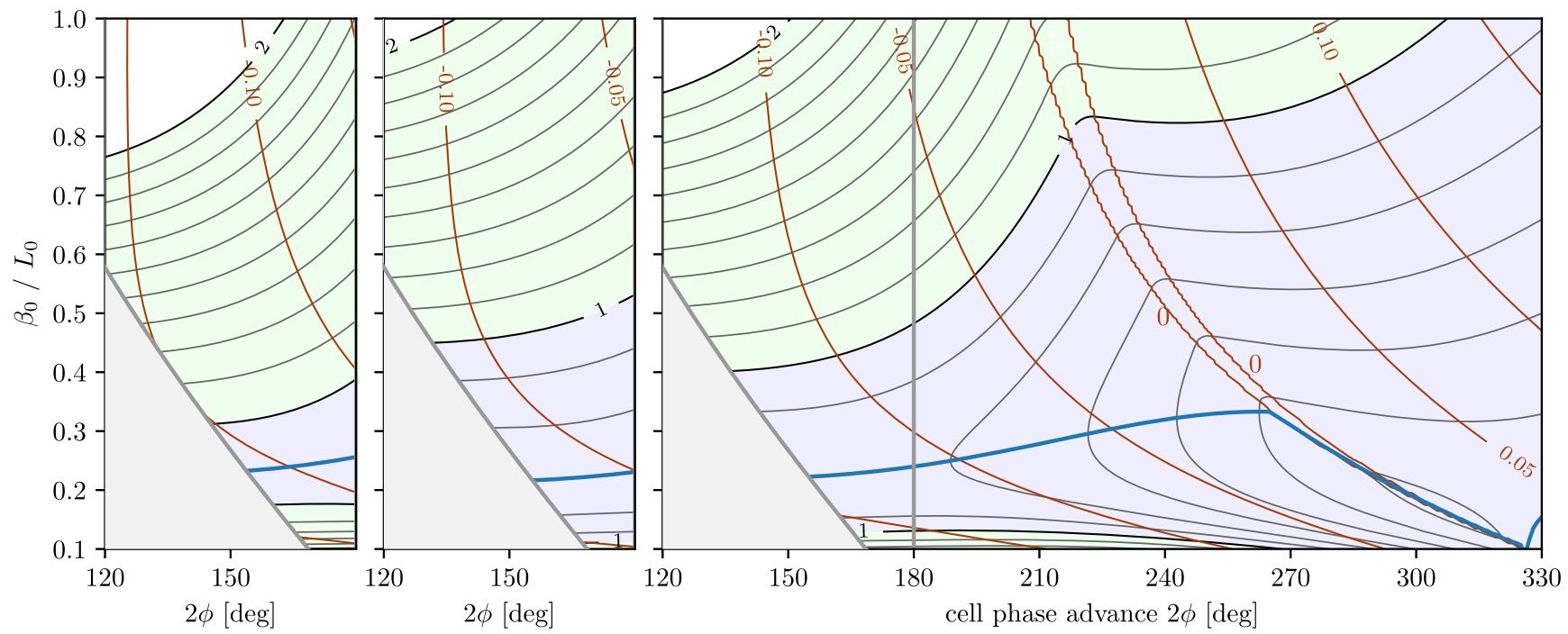

FIG. 15. Emittance ratio $F$ for LGB/RB cells with IDM magnet at $R=2$ (see legend in Fig. 13) with different values of $\beta_{1}$ at the reverse-bend center. From left to right: $\beta_{1}=20 L_{0}, \beta_{1}=40 L_{0}, \beta_{1}=30 L_{0}$. 
simultaneously using HOM, LGB $(b \geq 0)$, and HOM/RB cells, and that the LGB/RB cell type (as a generalization of IDM/RB cells) yields superior performance to the other investigated cell types. To apply further boundary conditions on the cell design, we need to assume specific properties on the quadrupole array in the cell interior (see also Appendix $\mathrm{C}$ for an elementary procedure using thin lenses).

\section{F. LGB/RB and IDM/RB example cells}

With an RB providing the means to suppress the dispersion (and with it the dispersion action $\mathcal{H}$ ) at the main bend center, optimization of the field variation now efficiently suppresses the fifth radiation integral by pushing the central field peak to very high values (LGB/RB example, Fig. 16). LGB profile and RB angle were optimized in common, since a high field peak calls for central dispersion close to zero, resulting in $\theta_{1}=-0.275^{\circ}$. At unchanged tunes $\phi, \phi_{\mathrm{y}}$, the emittance is reduced to $165 \mathrm{pm}$ (or $F=1.36$ ), which is only $36 \%$ of the HOM cell example (Sec. III C).
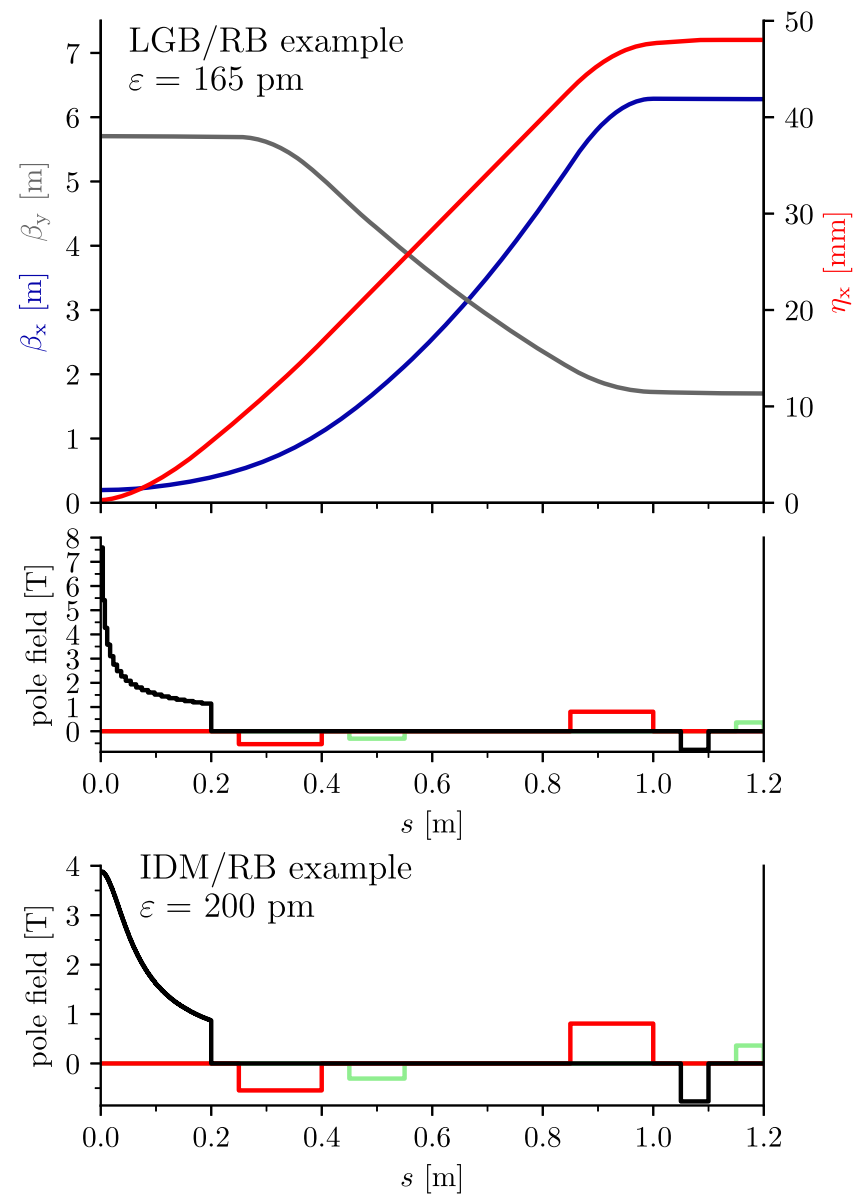

FIG. 16. Top: Field variation in a longitudinal gradient bend efficiently exploits the suppression of dispersion enabled by the reverse bend (see Fig. 4 for legend.) Bottom: Magnetic field of an LGB/RB example cell utilizing an IDM with $R=2$.
The problem of large field enhancement (see Sec. V E) is partially circumvented by using an IDM as main bend (IDM/RB example, Fig. 16). The emittance $\varepsilon=200 \mathrm{pm}$ (or $F=1.65$ ) is still only $45 \%$ of the value for the HOM cell-actually this ratio is smaller than the product of emittance ratios using only LGB without RB (92\%, Sec. III C) and using only RB without LGB (HOM/RB, $72 \%$, Sec. V D). Still, a moderate field enhancement factor $R=2$ corresponds to a peak field $B_{\max } \approx 4 \mathrm{~T}$ in our example cell.

As $B_{\max } \propto 1 / L_{0}$ for fixed field enhancement, the main bend length would need to be doubled to obtain similar emittances using a normal-conducting magnet, also beneficially reducing $\beta_{0}<L_{0} / 2$. To maintain the focusing constraints of the considered example cell, the main bend would be required to spatially overlap with the adjacent vertically focusing quadrupole.

\section{THE SLS 2.0 UNIT CELL}

In the previous sections, unit cells made of separatefunction magnets have been considered, optimizing the emittance using the radiation integrals $I_{5}$ (which only affects transverse emittance) and $I_{2}$.

In this section, we release the constraint of using separate-function magnets, which was required for our simplified dispersion matching and emittance model (where $\beta(s)$ in bending magnets propagates like in a drift space). This opens up the possibility of further manipulating the emittance via the horizontal damping partition $J_{\mathrm{x}}=1-I_{4} / I_{2}$, as is explained in more detail e.g. in $[1,6,14]$. The emittance then proportional to

$$
F \propto \frac{I_{5}}{I_{2} J_{\mathrm{x}}}=\frac{I_{5}}{I_{2}-I_{4}} \text { with } I_{4}=\int \eta b\left(b^{2}+2 k\right) \mathrm{d} s,
$$

where $k=\mathrm{d} b / \mathrm{d} x$ is the normalized transverse gradient, $k>0$ resulting in horizontal focusing.

It is thus possible to decrease $F$ by introducing simultaneous bending and focusing so that $\eta b \cdot k<0$. For positive dispersion, this requires vertical focusing in the main bend and horizontal focusing in the reverse bend; conveniently the gradients perform the function of a quadrupole doublet as required for focusing at sensible phase advances $2 \phi<\pi$.

In addition, the combination of the LGB with a quadrupole effectively increases $L_{0}$ and thus allows high field enhancement at lower absolute curvatures (see Sec. V F). The transverse gradient is included near the magnet ends with lower curvatures, where it is technically feasible.

Eventually, a real lattice cell based on the aforementioned combined-function magnets is shown in Fig. 17: it is the unit cell of the new storage ring for the upgrade of the Swiss Light Source SLS, named "SLS 2.0" [16-18]. Here the tunes were slightly shifted to $Q_{x}=0.4285 \approx 3 / 7$ and $Q_{y}=0.1429 \approx 1 / 7$ for optimal cancellation of sextupole and octupole resonances over an arc made of seven 


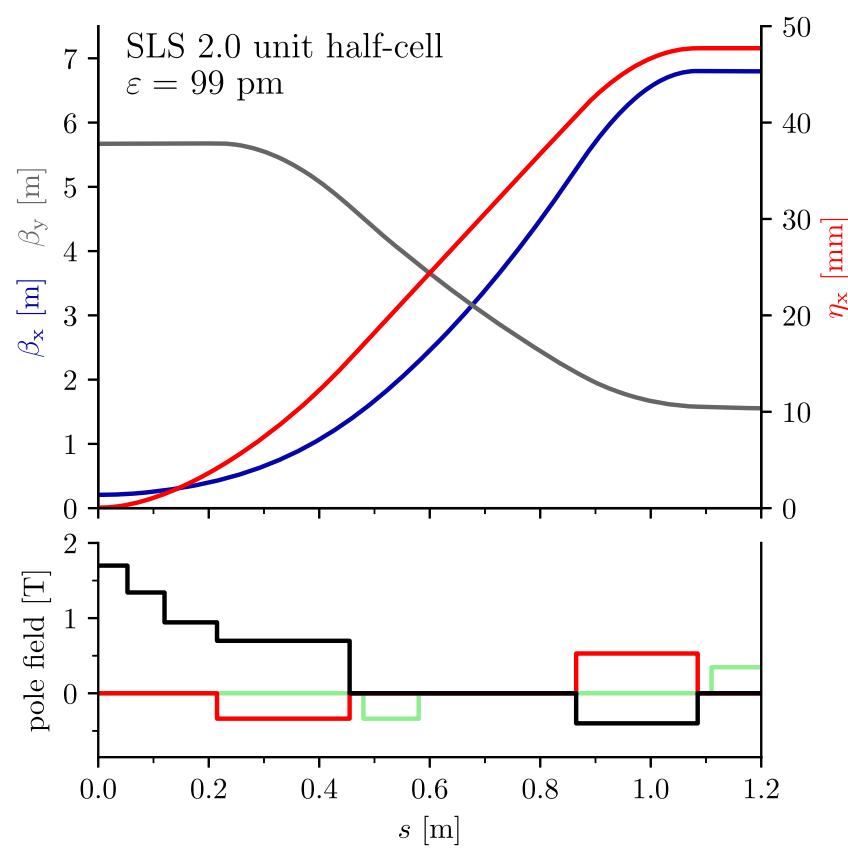

FIG. 17. The unit cell of the SLS upgrade lattice "SLS 2.0" represents a fully optimized, real LGB/RB cell (see Fig. 4 for legend).

cells [30]. The RB is merged with the horizontally focusing quadrupole - essentially the RB is a horizontally shifted quadrupole. The LGB is a permanent magnet with moderate peak field. The low field in the end pieces provides margin to introduce a transverse gradient for vertical focusing. The bending angles are $\theta_{0}=1.93^{\circ}+1.2^{\circ}$ (central half LGB + end piece) and $\theta_{1}=-0.63^{\circ}(\mathrm{RB})$, giving $5^{\circ}$ deflection for the complete unit cell.

Since time-of-flight effects in main and reverse bends compensate to some extent, LGB/RB cells become almost isochronous when tuned to the minimum emittance for given phase advance, which to some extent could be an attractive feature for damping rings. In light sources, however, longer bunches are required to provide adequate beam lifetime and instability thresholds. Therefore the RB angle in the SLS 2.0 cell is increased beyond its optimum value in order to realize a sufficiently large negative momentum compaction factor while accepting the emittance to be $9 \%$ larger than its possible minimum value.

A comparison of $I_{2}$ and $I_{5}$ radiation integrals for all considered example cells is shown in Fig. 18. The fraction of radiation integrals $I_{5} / I_{2}$ of the SLS 2.0 unit cell is located between that of the LGB/RB and IDM/RB examples. Both radiation integrals are lower in the SLS 2.0 case, since the fields on average are lower due to the increased effective length $L_{0}$ of the main bend. Thus, in comparison to the example cells, the major emittance reduction in terms of $I_{5} / I_{2}$ is due to reduction of $I_{5}$, which is beneficial as this integral only affects transverse emittances.

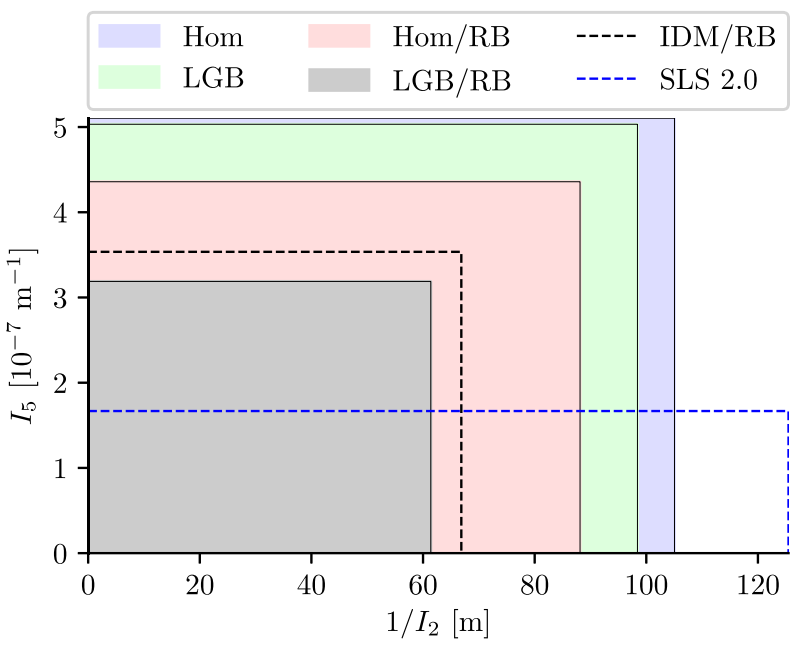

FIG. 18. Comparison of radiation integrals $I_{5}$ and $I_{2}$ for the example half-cells including SLS 2.0. The area of the respective rectangles is proportional to the emittance when approximating $J_{\mathrm{x}}=1$.

The final emittance of this unit cell is further reduced through the increased damping partition number $J_{\mathrm{x}}$. A positive transverse gradient in the $\mathrm{RB}$ and a negative gradient in the main bend both shift the damping partition in favor of the horizontal dimension resulting in $J_{\mathrm{x}}=$ 1.795, whereas $\left|J_{\mathrm{x}}-1\right|<2 \times 10^{-3}$ in all previous example cases (see drift-space assumption, Sec. II).

After application of all described optimizations and design choices, a unit cell emittance of $\varepsilon=99 \mathrm{pm}$ is obtained for a beam energy of $2.4 \mathrm{GeV}$, which corresponds to a TME emittance ratio $F=0.82$.

\section{CONCLUSION}

1. We reviewed the commonly known fact that the TME condition is not realized in MBA unit cells due its high phase advance $2 \phi>\pi$. Instead, HOM cells with sensible phase advances $2 \phi<\pi$ are used. The resulting optical mismatch in the bend causes an emittance increase of such cells $(F>2.45) .2$. We demonstrated that LGB shapes [9] exist which provide significant emittance reduction at their optimal, very high phase advances $\phi>\phi_{\mathrm{TME}}$, but which actually provide only marginal emittance reduction compared to a a HOM cell for sensible phase advances. Even without specification of any particular shape, there seems to exist a principal limit $F>2$ of achievable emittance reduction by LGBs for sensible phase advances. 3. Freeform optimization of LGB curvatures without constraints on curvature polarity results in two regimes: positive curvatures in the magnet center and negative curvature at its ends, i.e., the bend naturally splits up into a main bend and a reverse bend. 4 . The concept of the reverse-bend cell [14] has been revisited. It has been shown that for sensible phase advances, the reverse-bend cell is able to provide significantly lower emittances than HOM cells through 
suppression of dispersion in the main bend center $(F<2$ is possible for $2 \phi<\pi)$. 5 . When combining a reverse bend with an LGB (LGB/RB cell), its potential in emittance reduction can be fully exploited and even lower emittance values $F<1$ are also possible for sensible phase advances $2 \phi<\pi$ : a central peak of high field concentrates most bending in the region of suppressed dispersion, while decay of field strength toward the edges compensates for the inevitable growth of dispersion, thus minimizing the quantum excitation integral.

\section{ACKNOWLEDGMENTS}

We would like to thank our colleagues M. Aiba, M. Böge, J. Chrin, T. Schietinger, and J. Kallestrup, as well as J. Bengtsson (DIAMOND) for discussions, proofreading, and useful comments on this work.

\section{APPENDIX A: EXAMPLE CELL CHARACTERISTICS}

All example half-cells but the SLS 2.0 cell start with the following sequence (1) main half-bend (length $L_{0}=0.2 \mathrm{~m}$, bending angle $\theta_{0}=2.5^{\circ}$ ). (2) drift space (length $0.05 \mathrm{~m}$ ). (3) defocusing quadrupole (length $0.15 \mathrm{~m}$, focusing strength $k_{\mathrm{D}}$ ). (4) drift space (length $0.45 \mathrm{~m}$ ). This space is sufficient for a defocusing sextupole magnet. In our example, the magnet starts $0.05 \mathrm{~m}$ from the beginning of the drift and is $0.1 \mathrm{~m}$ long. (5) focusing quadrupole (length $0.15 \mathrm{~m}$, focusing strength $k_{\mathrm{F}}$ ).

If no reverse bend is included, the sequence is concluded with (6a) drift space (length $0.1 \mathrm{~m}$ ). This drift space is sufficient for a focusing sextupole with $0.1 \mathrm{~m}$ length, centered in the symmetry point. The total length of this half-cell is $1.1 \mathrm{~m}$. For the HOM cell, the main bend is homogeneous by definition. For the LGB cell, the optimized main bend can be described in good approximation by the field

$$
B(s)=2.695 \mathrm{~T}-\sqrt{0.1 \mathrm{~T}^{2}+75(\mathrm{~T} / \mathrm{m})^{2} s^{2}} .
$$

With a reverse bend, the mentioned main half-bend is modified so that its angle is $\theta_{0}-\theta_{1}$. The sequence

TABLE I. Focusing strengths $k$, natural chromaticities $\xi_{\text {nat }}$, and required integrated sextupole strengths $m$ to compensate chromaticities to zero, for four example cells.

\begin{tabular}{lrrrr}
\hline \hline & HOM & LGB & HOM/RB & IDM/RB \\
\hline$k_{\mathrm{D}}\left[\mathrm{m}^{-2}\right]$ & -5.2707 & -5.3384 & -5.1781 & -5.2445 \\
$k_{\mathrm{F}}\left[\mathrm{m}^{-2}\right]$ & 7.7810 & 7.7860 & 7.7528 & 7.7567 \\
$\xi_{\text {nat }}^{\mathrm{n}}$ & -1.0908 & -1.0899 & -1.0090 & -1.0046 \\
$\xi_{\text {nat }}^{\mathrm{y}}$ & -0.3063 & -0.3063 & -0.3418 & -0.3427 \\
$m_{\mathrm{D}}\left[\mathrm{m}^{-2}\right]$ & -20.300 & -20.291 & -21.807 & -22.624 \\
$m_{\mathrm{F}}\left[\mathrm{m}^{-2}\right]$ & 13.009 & 13.000 & 13.288 & 13.409 \\
\hline \hline
\end{tabular}
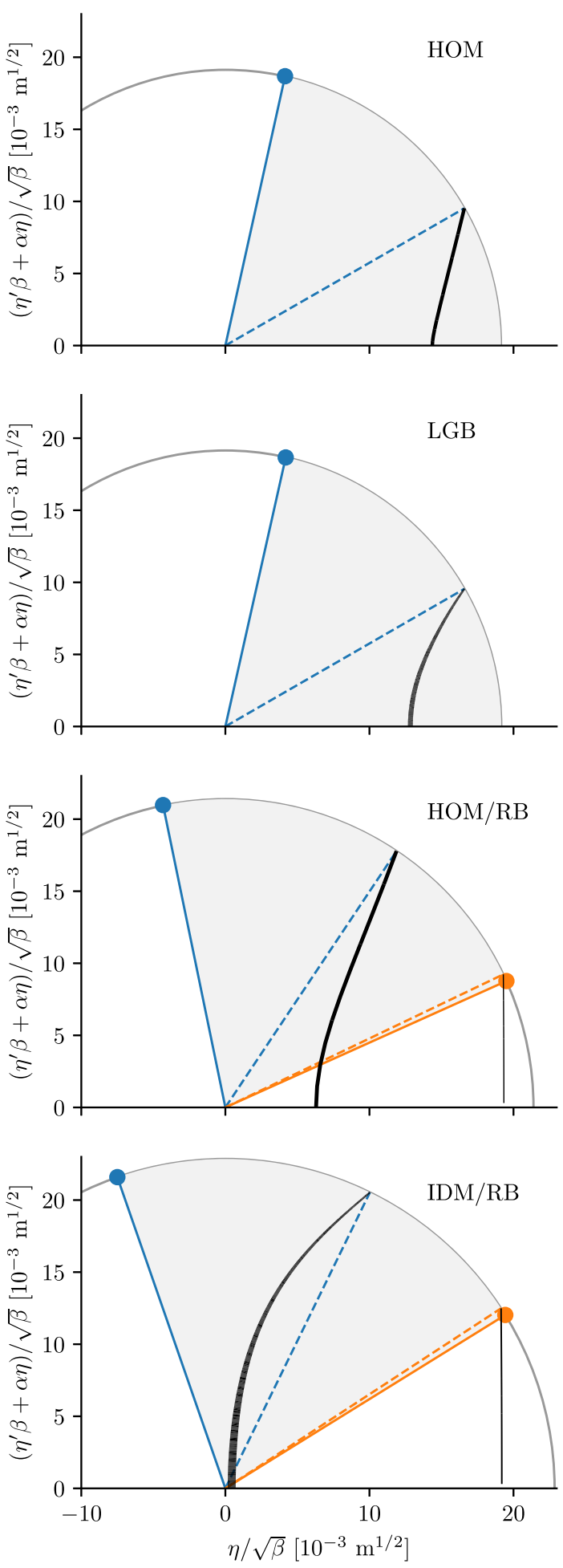

FIG. 19. Dispersion in normalized phasespace (black lines) for four example cells; the line thickness corresponds to local field strength. Markers indicate $\vec{P}_{0}$ (blue) and $\vec{P}_{1}$ (orange). Dashed lines mark the betatron phase at the exit of the main bend (blue) and the entrance of the reverse bend (orange). Thus the angle between solid and dashed line of the same color is the phase advance inside the respective half-bend. The angle included in the shaded arc (limited by $\vec{P}_{0}, \vec{P}_{1}$ ) is the half-cell phase advance $\phi\left(77.4^{\circ}\right.$ for shown examples). 
continues as $(6 \mathrm{~b})$ drift space (length $0.05 \mathrm{~m}$ ). (7) reverse half-bend (length $L_{1}=0.05 \mathrm{~m}$, bending angle $\theta_{1}=-0.2^{\circ}$ ). (8) drift space (length $0.1 \mathrm{~m}$ ). As this is the last element of the sequence, the drift space is sufficient to install a focusing sextupole with $0.1 \mathrm{~m}$ length, centered in the symmetry point. The half-length of such a cell is $1.2 \mathrm{~m}$. While the main bend of the HOM/RB example cell is again homogeneous, the main bend of the IDM/RB example cell obviously is an IDM with field enhancement factor $R=2$ (Sec. IV B).

The parameters and resulting natural chromaticities of four example cells are listed in Table I. Figure 19 shows their dispersion trajectories in the normalized phase space.

\section{APPENDIX B: EMITTANCE COEFFICIENTS FOR MAGNETS}

With the drift-space assumption $\beta(s)=\beta_{0}+s^{2} / \beta_{0}$ and Eq. (2), and omitting the indices of $L_{q}, \theta_{q}$ for convenience, the normalized dispersion action of Eqs. (12) and Eq. (13) evaluates to (see [9])

$$
\begin{aligned}
\hat{\mathcal{H}} & =\frac{H}{L \theta^{2}} \\
& =\frac{L}{\beta_{q}}\left[\left(\frac{\eta_{q}}{\theta L}\right)^{2}-2 \frac{\eta_{q}}{\theta L} v(s)+v^{2}(s)\right]+\frac{\beta_{q}}{L}\left(\frac{\eta^{\prime}(s)}{\theta}\right)^{2} .
\end{aligned}
$$

where we introduced the lever function

$$
v(s)=\frac{1}{\theta L}\left(s \eta^{\prime}(s)-\int_{0}^{s} \eta^{\prime}(\tilde{s}) \mathrm{d} \tilde{s}\right)=\frac{1}{\theta L} \int_{0}^{s} \tilde{s} b(\tilde{s}) \mathrm{d} \tilde{s} .
$$

Multiplication of Eq. (B1) and averaging results in $\left\langle\left|\hat{b}^{3}\right| \hat{\mathcal{H}}\right\rangle$. The prefactor of $\beta_{q} / L$ modifies to

$$
C=\left\langle\left|\hat{b}^{3}\right|\left(\frac{\eta^{\prime}}{\theta}\right)^{2}\right\rangle
$$

while the prefactor of $L / \beta_{q}$ modifies to

$$
\left\langle\left|\hat{b}^{3}\right|\right\rangle\left(\frac{\eta_{0}}{\theta L}\right)^{2}-2\left\langle\left|\hat{b}^{3}\right| v\right\rangle\left(\frac{\eta_{0}}{\theta L}\right)+\left\langle\left|\hat{b}^{3}\right| v^{2}\right\rangle .
$$

These remaining four averages in $\left\langle\left|\hat{b}^{3}\right| \hat{\mathcal{H}}\right\rangle$ only depend on $\hat{b}(s)$.

To furthermore compute the phase advance for any magnet, a relation between $\eta_{\vee}$ and $\eta_{0}$ (or $\eta_{\wedge}$ and $\eta_{1}$ ) is established by the thin-dipole dispersion difference

$$
\eta_{0}=\eta_{\vee}+V \theta_{0} L_{0} \quad \text { with } \quad V=v\left(L_{0}\right) .
$$

For magnets with positive orbit curvature, $b, \eta^{\prime}, v$ are all positive; in this case, the lever function and its final value are related as $V=v\left(L_{0}\right)=\max v(s)$. For Eq. (28) positive curvature thus implies

$$
\tilde{A}=2\left(\left\langle\left|\hat{b}^{3}\right|\right\rangle V-\left\langle\left|\hat{b}^{3}\right| v\right\rangle\right)=2\left\langle\left|\hat{b}^{3}\right|(V-v)\right\rangle>0 .
$$

\section{Homogeneous magnet}

For this magnet type, extending from $0<s<L$,

$$
\hat{b}(s)=1, \quad \frac{\eta^{\prime}(s)}{\theta}=\frac{s}{L}, \quad v(s)=\frac{1}{2}\left(\frac{s}{L}\right)^{2} .
$$

Note that the sign of bending angle is normalized out of $\hat{b}$, so that it can also be used for reverse bends. This results in

$$
\begin{aligned}
\left\langle\left|\hat{b}^{3}\right|\right\rangle=1, \quad & \left\langle\hat{b}^{3} \mid v\right\rangle=\frac{1}{6}, \quad\left\langle\left|\hat{b}^{3}\right| v^{2}\right\rangle=\frac{1}{20}, \\
\left\langle\hat{b}^{2}\right\rangle & =1, \quad V=\frac{1}{2}, \quad C=\frac{1}{3} .
\end{aligned}
$$

\section{Inverse-distance scaling magnet (IDM)}

Using the inverse distance $w(s)=\sqrt{1+(s / h)^{2}}$, the dispersion-related functions of the IDM are obtained as

$$
\begin{aligned}
\frac{\eta^{\prime}(s)}{\theta} & =\frac{\operatorname{arsinh}(s / h)}{\operatorname{arsinh}(L / h)}, \quad v(s)=R(h / L)^{2}(w(s)-1), \\
V & =R(h / L)^{2}(W-1) \quad \text { with } \quad W=w(L) .
\end{aligned}
$$

Most emittance coefficients can be expressed via averages of the form $\left\langle w^{-m}\right\rangle$ with positive integer $m$,

$$
\begin{aligned}
\left\langle\hat{b}^{2}\right\rangle & =R^{2}\left\langle w^{-2}\right\rangle, \quad\left\langle\left|\hat{b}^{3}\right|\right\rangle=R^{3}\left\langle w^{-3}\right\rangle, \\
\left\langle\left|\hat{b}^{3}\right| v\right\rangle & =R^{4}(h / L)^{2}\left(\left\langle w^{-2}\right\rangle-\left\langle w^{-3}\right\rangle\right), \\
\left\langle\left|\hat{b}^{3}\right| v^{2}\right\rangle & =R^{5}(h / L)^{4}\left(\left\langle w^{-1}\right\rangle-2\left\langle w^{-2}\right\rangle+\left\langle w^{-3}\right\rangle\right) .
\end{aligned}
$$

The case $m=1$ of this average evaluates to $\left\langle w^{-1}\right\rangle=$ $\operatorname{arsinh}(L / h) \cdot h / L$. For larger $m$, one may use the substitution $u=\arctan (s / h)$ so that the integrand transforms to $\cos ^{m-2} u$; the integral is then solved by recursive application of the cosine reduction formula.

The remaining coefficient for the emittance is

$$
C=\frac{R^{3}}{\operatorname{arsinh}^{2}(L / h)}\left\langle\frac{\operatorname{arsinh}^{2}(s / h)}{w^{3}}\right\rangle .
$$


Introducing the abbreviation $a=\operatorname{arsinh}(L / h)$, the average $\left\langle\operatorname{arsinh}^{2}(s / h) / w^{3}\right\rangle$ evaluates to

$\frac{h}{L}\left(\operatorname{dil} \log \left(1+\mathrm{e}^{-2 a}\right)+a^{2} \frac{L / h-W}{W}-2 a \ln \left(1+\mathrm{e}^{-2 a}\right)+\frac{\pi^{2}}{12}\right)$

with the dilogarithm dilog $z=\int_{0}^{z} \frac{\ln t}{1-t} \mathrm{~d} t$.

\section{Free-form LGB shapes}

For numerical integration, we split $b(s)$ into a piece-wise constant function with $Q$ pieces

$$
\hat{b}(s)=\underline{b}_{q} \text { for } s_{q-1}<s<s_{q},
$$

with $s_{0}=0, s_{Q}=L$, and the values $s_{q}$ being equidistant with $\Delta s=L / Q$. In that case, $\eta^{\prime}(s)$ is a piece-wise linear function with

$$
\frac{\eta^{\prime}\left(s_{p}\right)}{\theta}=\frac{1}{Q} \sum_{q=1}^{p} \underline{b}_{q}
$$

being a cumulative sum $(0<p \leq Q)$. The lever function Eq. (B2) follows as

$$
v\left(s_{p}\right)=\frac{1}{Q} \sum_{q=1}^{p} \frac{s_{q}+s_{q-1}}{2 L} \underline{b}_{q}
$$

The emittance coefficients have more complicated dependencies on the $\underline{b}_{q}$ values, and one may compute them using the numerical average in good approximation, subsequently calculating $F$ using Eq. (28).

Note that all described calculations (also including the normalization requirement $\eta^{\prime}(L)=\theta$ ) are differentiable transformations of the parameters $\underline{b}_{q}$, which allows us to compute the gradient of the discretized functional $\mathrm{d} F / \mathrm{d} \underline{b}_{q}$ for all $q$.

\section{APPENDIX C: MATCHING THE BEND-FREE REGION FOR GIVEN PARAMETERS}

Of the four free parameters of a reverse-bend cell $\phi, \beta_{0}$, $\beta_{1}, \theta_{1} / \theta_{0}$, the former three parameters define the half-cell transfer matrix $\mathbf{T}$ via Eq. (4), and the ratio $\theta_{1} / \theta_{0}$ defines the magnet lengths $L_{0}, L_{1}$ via Eq. (44). The magnet transfer matrices are assumed as drift spaces $\mathbf{D}_{0}, \mathbf{D}_{1}$. In consequence, the horizontal transfer matrix of the bend-free region can be calculated as

$$
\begin{aligned}
\mathbf{M} & =\mathbf{D}_{1}^{-1} \mathbf{B}_{1} \mathbf{R}(\phi) \mathbf{B}_{0}^{-1} \mathbf{D}_{0}^{-1} \\
& =\frac{1}{\sqrt{\beta_{0} \beta_{1}}}\left(\begin{array}{cc}
\beta_{1} & -L_{1} \\
0 & 1
\end{array}\right) \mathbf{R}(\phi)\left(\begin{array}{cc}
1 & -L_{0} \\
0 & \beta_{0}
\end{array}\right) .
\end{aligned}
$$

To obtain a half-cell for given $\phi, \beta_{0}, \beta_{1}$, the transfer matrix $\mathbf{M}$ of the bend-free region must thus be matched by a given array of quadrupoles, while also guaranteeing vertical stability. This naturally requires two or more quadrupoles, separated by drift spaces.

The conceptually simplest example is a Galilean telescope, modeled using two thin lenses with focal lengths of different signs $f_{0}, f_{1}$ directly attached to the ends of the bending magnets, and an intermediate drift space of length $d$. As the transfer matrix of this telescope must be $\mathbf{M}$, one obtains

$d=M_{12}, \quad \frac{d}{f_{0}}=1-M_{11}, \quad \frac{d}{f_{1}}=1-M_{22}$.

The sign of $f_{0}$ is given by the calculation of $M_{11}$ from Eq. (C1)

$$
M_{11}=\sqrt{\frac{\beta_{1}}{\beta_{0}}} \cos \phi+\frac{L_{1}}{\sqrt{\beta_{0} \beta_{1}}} \sin \phi .
$$

For the interesting range of phase advances $\phi<\pi / 2$ and under the assumption $\beta_{1} / \beta_{0} \gg 1$, we conclude that the focal length of the main-bend lens $f_{0}$ is negative.

[1] D. Einfeld and M. Plesko, A modified QBA optics for low emittance storage rings, Nucl. Instrum. Methods Phys. Res., Sect. A 335, 402 (1993).

[2] D. Einfeld, Multi-bend achromat lattices for storage ring light sources, Synchrotron Radiat. News 27, 4 (2014).

[3] L. Farvacque, N. Carmignani, J. Chavanne, A. Franchi, G. L. Bec, S. Liuzzo, B. Nash, T. Perron, and P. Raimondi, in Proceedings of the 4th International Particle Accelerator Conference, IPAC-2013, Shanghai, China, 2013 (JACoW, Shanghai, China, 2013), p. 79, https://accelconf.web.cern .ch/AccelConf/IPAC2013/papers/mopea008.pdf.

[4] A. F. Wrulich, Overview of third generation light sources, in Proceedings of Workshop on Fourth Generation Light Sources, Report No. SSRL 92/02, 1992, http://inspirehep .net/record/334923.

[5] S. C. Leemann, A. Andersson, M. Eriksson, L.-J. Lindgren, E. Wallén, J. Bengtsson, and A. Streun, Beam dynamics and expected performance of Sweden's new storage-ring light source: MAX IV, Phys. Rev. ST Accel. Beams 12, 120701 (2009).

[6] H. Wiedemann, Particle Accelerator Physics, 4th ed. (Springer, New York, 2015).

[7] S. C. Leemann and A. Streun, Perspectives for future light source lattices incorporating yet uncommon magnets, Phys. Rev. ST Accel. Beams 14, 030701 (2011).

[8] L.C. Teng, Minimum emittance lattice for synchrotron radiation storage rings, Argonne National Laboratory Technical Report No. LS-17, 1985.

[9] A. Streun and A. Wrulich, Compact low emittance light sources based on longitudinal gradient bending magnets, Nucl. Instrum. Methods Phys. Res., Sect. A 770, 98 (2015). 
[10] M. A. Domínguez Martinez, F. Toral, H. Ghasem, P. S. Papadopoulou, and Y. Papaphilippou, Longitudinally variable field dipole design using permanent magnets for CLIC damping rings, IEEE Trans. Appl. Supercond. 28, 1 (2018).

[11] R. Nagaoka and A.F. Wrulich, Emittance minimisation with longitudinal dipole field variation, Nucl. Instrum. Methods Phys. Res., Sect. A 575, 292 (2007).

[12] K. Steffen, The Wiggler Storage Ring: A device with strong radiation damping and small beam emittance, DESY Technical Report No. PET-79/05, 1979.

[13] J. P. Delahaye and J. P. Potier, Reverse bending magnets in combined-function lattice for the CLIC damping ring, in Proceedings of the 1989 Particle Accelerator Conference, Chicago, IL (IEEE, New York, 1989), p. 1611, http:// accelconf.web.cern.ch/AccelConf/p89/PDF/ PAC1989_1611.PDF.

[14] A. Streun, The anti-bend cell for ultralow emittance storage ring lattices, Nucl. Instrum. Methods Phys. Res., Sect. A 737, 148 (2014).

[15] OPA accelerator optics software with examples, http://ados .web.psi.ch/opa (2017).

[16] A. Streun, T. Garvey, L. Rivkin, V. Schlott, T. Schmidt, P. Willmott, and A. Wrulich, SLS-2 - the upgrade of the Swiss Light Source, J. Synchrotron Radiat. 25, 631 (2018).

[17] M. Aiba et al., SLS-2 Conceptual Design Report, edited by A. Streun (Paul Scherrer Institut, 2017), http://www.lib4ri .ch/archive/nebis/PSI_Berichte_000478272/PSIBericht_17-03.pdf.

[18] M. Aiba, M. Böge, T. Garvey, V. Schlott, and A. Streun, Towards an upgrade of the Swiss light source, in Proceedings of 9th International Particle Accelerator Conference (IPAC'18), Vancouver (JACoW, Geneva, Switzerland 2018), p. 4358, https://doi.org/10.18429/JACoWIPAC2018-THPMK029.

[19] D. Trbojevic and E. Courant, in Proceedings of the Fourth European Particle Accelerator Conference EPAC 94, London, England (World Scientific, River Edge, NJ,
1994), p. 1000, http://accelconf.web.cern.ch/AccelConf/ e94/PDF/EPAC1994_1000.PDF.

[20] C.-x. Wang, Minimum emittance in storage rings with uniform or nonuniform dipoles, Phys. Rev. ST Accel. Beams 12, 061001 (2009).

[21] K. Wille, The Physics of Particle Accelerators (Oxford University Press, New York, 2000).

[22] M. Sands, The physics of electron storage rings: An introduction, SLAC Technical Report No. R-121, 1979, with addendum.

[23] J. Tanabe, Iron dominated electromagnets design, fabrication, assembly and measurements, SLAC Technical Report No. R-754, 2005.

[24] A. Streun, M. Aiba, M. Böge, M. Ehrlichman, and Á. Saá Hernández, Design studies for an upgrade of the SLS storage ring, in Proceedings of 6th International Particle Accelerator Conference (IPAC'15), Richmond (JACoW, Geneva, Switzerland, 2015) p. 1724, https://doi.org/10 .18429/JACoW-IPAC2015-TUPJE047.

[25] C.-x. Wang, Y. Wang, and Y. Peng, Optimal dipole-field profiles for emittance reduction in storage rings, Phys. Rev. ST Accel. Beams 14, 034001 (2011).

[26] D. Maclaurin, D. Duvenaud, M. Johnson, J. Townsend et al., autograd, https://github.com/HIPS/autograd (2018).

[27] D. Maclaurin, Modeling, inference and optimization with composable differentiable procedures, Ph.D. thesis, Harvard University, 2016.

[28] C. Zhu, R. H. Byrd, P. Lu, and J. Nocedal, Algorithm 778: L-BFGS-B: Fortran subroutines for large-scale boundconstrained optimization, ACM Trans. Math. Softw. 23, 550 (1997).

[29] T. Zhang and X. Huang, Numerical optimization of a low emittance lattice cell, Nucl. Instrum. Methods Phys. Res., Sect. A 923, 55 (2019).

[30] J. Bengtsson and A. Streun, Robust Design Strategy for SLS-2, Paul Scherrer Institut Technical Report No. SLS2BJ84-001, 2017. 